\title{
2006 WHOLE EARTH TELESCOPE OBSERVATIONS OF GD358: A NEW LOOK AT THE PROTOTYPE DBV
}

J. L. Provencal ${ }^{1,2}$, M. H. Montgomery ${ }^{2,3}$, A. KanaAn ${ }^{4}$, H. L. Shipman ${ }^{1}$, D. Childers ${ }^{5}$, A. Baran ${ }^{6}$, S. O. KePler $^{7}$, M. Reed $^{8}$,

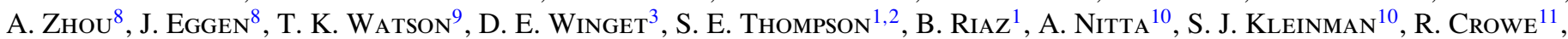
J. SlivkofF ${ }^{11}$, P. Sherard ${ }^{11}$, N. Purves ${ }^{11}$, P. Binder ${ }^{11}$, R. Knight ${ }^{11}$, S. -L. Kim ${ }^{12}$, Wen-Ping CheN ${ }^{13}$, M. Yang ${ }^{13}$, H. C. Lin ${ }^{13}$, C. C. Lin $^{13}$, C. W. Chen ${ }^{13}$, X. J. Jiang ${ }^{14}$, A. V. Sergeev ${ }^{15}$, D. Mkrtichian ${ }^{16,17}$, M. Andreev ${ }^{15}$, R. Janulis ${ }^{18}$, M. SiwaK ${ }^{19}$, S. Zola ${ }^{6,19}$, D. Koziel ${ }^{19}$, G. Stachowski ${ }^{6}$, M. PaPAro ${ }^{20}$, Zs. Bognar ${ }^{20}$, G. HANdler ${ }^{21}$, D. Lorenz ${ }^{21}$, B. Steininger ${ }^{21}$, P. BeCK ${ }^{21}$, T. NAGEL ${ }^{22}$, D. Kusterer ${ }^{22}$, A. HofFMAN ${ }^{22}$, E. ReifF ${ }^{22}$, R. KowAlski ${ }^{22}$, G. VAuClair ${ }^{23}$, S. CHARPINET $^{23}$, M. Chevreton ${ }^{24}$, J. E. Solheim ${ }^{25}$, E. Pakstiene ${ }^{26}$, L. Fraga ${ }^{4}$, And J. Dalessio ${ }^{1,2}$

${ }^{1}$ University of Delaware, Department of Physics and Astronomy, Newark, DE 19716, USA; jlp@udel.edu

${ }^{2}$ Delaware Asteroseismic Research Center, Mt. Cuba Observatory, Greenville, DE 19807, USA

${ }^{3}$ Department of Astronomy, University of Texas, Austin, TX-78712, USA; mikemon@ rocky.as.utexas.edu

${ }^{4}$ Departamento de Física Universidade Federal de Santa Catarina, C.P. 476, 88040-900, Florianópolis, SC, Brazil; ankanaan@gmail.com

${ }^{5}$ Department of Math and Science, Delaware County Community College, 901 S. Media Rd, Media, PA-19063, USA; dpc@udel.edu

${ }^{6}$ Mount Suhora Observatory, Cracow Pedagogical University, Ul. Podchorazych 2, 30-084 Krakow, Poland; zola@astro1.as.ap.krakow.pl

${ }^{7}$ Instituto de Fíisica UFRGS, C.P. 10501, 91501-970 Porto Alegre, RS, Brazil; kepler@if.ufrgs.br

${ }^{8}$ Missouri State University and Baker Observatory, 901S. National, Springfield, MO 65897, USA; MikeReed@missouristate.edu

${ }^{9}$ Southwestern University, Georgetown, TX, USA; tkw@southwestern.edu

${ }^{10}$ Gemini Observatory, Northern Operations Center, 670 North A'ohoku Place, Hilo, HI 96720, USA; atsuko.nittakleinman@gmail.com ${ }^{11}$ University of Hawaii, Hilo, HI 96720, USA; rcrowe@hubble.uhh.hawaii.edu

12 Korea Astronomy and Space Science Institute, Daejeon 305-348, Korea; slkim@kasi.re.kr

${ }^{13}$ Lulin Observatory, National Central University, Taiwan; wchen@astro.ncu.edu.tw

${ }^{14}$ National Astronomical Observatories, Academy of Sciences, Beijing 100012, People’s Republic of China; xjjiang@bao.ac.cn

${ }^{15}$ Ukrainian National Academy of Sciences, Main Astronomical Observatory, Golosiiv, Kiev 022 252650, Ukraine; sergeev@terskol.com

${ }^{16}$ Astronomical Observatory, Odessa National University, Shevchenko Park, Odessa 65014, Ukraine; david@arcsec.sejong.ac.kr

${ }^{17}$ Astrophysical Research Center for the Structure and Evolution of the Cosmos, Sejong University, Seoul 143-747, Korea, davidm@sejong.ac.kr

${ }^{18}$ Institute of Theoretical Physics and Astronomy, Vilnius University, Vilnius, Lithuania; jr@itpa.lt

${ }^{19}$ Astronomical Observatory, Jagiellonian University, ul. Orla 171, 30-244 Cracow, Poland

${ }^{20}$ Konkoly Observatory, P.O. Box 67, H-1525 Budapest XII, Hungary; paparo@konkoly.hu

${ }^{21}$ Institut für Astronomie Universität Wien, Türkenschanzstrasse 17, 1180, Austria; handler@astro.univie.ac.at

${ }^{22}$ Institut für Astronomie und Astrophysik, Universiät Tübingen, Sand 1, 72076 Tübingen, Germany; nagel@astro.uni-tuebingen.de

${ }^{23}$ Laboratoire d'Astrophysique de Toulouse-Tarbes, Université de Toulouse, CNRS, 14 avenue Edouard Belin, F314000 Toulouse, France; gerardv@srvdec.obs-mip.fr

${ }^{24}$ Observatoire de Paris, LESIA, 92195 Meudon, France

${ }^{25}$ Institute of Theoretical Astrophysics, University of Oslo, P.O. Box 1029, Oslo, Norway; janerik@phys.uit.no

${ }^{26}$ Institute of Theoretical Physics and Astronomy, Astronomical Observatory, Gostauto 12, Vilnius LT 2600, Lithuania; erika@itpa.lt Received 2008 August 19; accepted 2008 November 4; published 2009 March 2

\begin{abstract}
We report on the analysis of $436.1 \mathrm{hr}$ of nearly continuous high-speed photometry on the pulsating DB white dwarf GD358 acquired with the Whole Earth Telescope (WET) during the 2006 international observing run, designated XCOV25. The Fourier transform (FT) of the light curve contains power between 1000 and $4000 \mu \mathrm{Hz}$, with the dominant peak at $1234 \mu \mathrm{Hz}$. We find 27 independent frequencies distributed in 10 modes, as well as numerous combination frequencies. Our discussion focuses on a new asteroseismological analysis of GD358, incorporating the 2006 data set and drawing on 24 years of archival observations. Our results reveal that, while the general frequency locations of the identified modes are consistent throughout the years, the multiplet structure is complex and cannot be interpreted simply as $l=1$ modes in the limit of slow rotation. The high- $k$ multiplets exhibit significant variability in structure, amplitude and frequency. Any identification of the $m$ components for the high- $k$ multiplets is highly suspect. The $k=9$ and 8 modes typically do show triplet structure more consistent with theoretical expectations. The frequencies and amplitudes exhibit some variability, but much less than the high- $k$ modes. Analysis of the $k=9$ and 8 multiplet splittings from 1990 to 2008 reveal a long-term change in multiplet splittings coinciding with the 1996 sforzando event, where GD358 dramatically altered its pulsation characteristics on a timescale of hours. We explore potential implications, including the possible connections between convection and/or magnetic fields and pulsations. We suggest future investigations, including theoretical investigations of the relationship between magnetic fields, pulsation, growth rates, and convection.
\end{abstract}

Key words: stars: evolution - stars: individual (GD358) - stars: oscillations - white dwarfs

\section{INTRODUCTION}

Asteroseismology of stellar remnants is traditionally the study of the interior structure of pulsating white dwarfs and subdwarfs as revealed by global stellar oscillations. The oscillations allow a view beneath the photosphere, and contain information about basic physical parameters, such as mass, rotation rate, internal transition profiles, and compositional structure. This information (see, for example, Nather et al. 1990; Winget et al. 1991; Winget et al. 1994; Kepler et al. 2000; Kanaan et al. 2005) provides important constraints on fields ranging from stellar formation and evolution, chemical evolution in our galaxy, the age of the galactic disk, the physics of Type Ia supernovae, and neutrino physics (Winget et al. 2004). 
Asteroseismology is now expanding its focus to attack problems that at first consideration may not seem best suited for the technique. Convection remains one of the largest sources of theoretical uncertainty in our understanding of stars. This lack of understanding leads to considerable systematic uncertainties in such important quantities as the ages of massive stars where convective overshooting is important (Di Mauro et al. 2003) and the temperatures and cooling ages of white dwarfs (Prada et al. 2002; Wood 1992). One important early result from the Canadian asteroseismology mission Microvariability and Oscillations of Stars (MOST) is the difficulty in detecting predicted oscillations in Procyon A, implying possibly serious incompleteness in our understanding of stars even slightly different from the Sun (Matthews et al. 2004; Marchenko 2008).

Montgomery (2005) shows how precise observations of variable star light curves can be used to characterize the convection zone in a pulsating star. Montgomery bases his approach on important analytical (Goldreich \& Wu 1999; Wu 2001) and numerical precursor calculations (Brickhill 1992). The method is based on three assumptions: (1) the flux perturbations are sinusoidal below the convection zone, (2) the convection zone is so thin that local angular variations of the nonradial pulsations may be ignored, i.e., we treat the pulsations locally as if they were radial, and (3) the convective turnover time is short compared with the pulsation timescale, so the convection zone can be assumed to respond instantaneously. Using high signal-to-noise light curves to model nonlinear effects, this approach can observationally determine the convective time scale $\tau_{0}$, a temperature dependence parameter $N$, and, together with an independent $T_{\text {eff }}$ determination, the classical convective efficiency parameter (the mixing length ratio) $\alpha$.

Montgomery (2005) applies this theoretical construct to two large amplitude, monoperiodic white dwarf variables where it is possible to fold long light curves to obtain high signal-to-noise pulse shapes. The test candidates are a hydrogen atmosphere DAV (G29-38) and a helium atmosphere DBV (PG1351+489). G29-38 is a well-studied DAV known for the complexity of its pulsations (Kleinman et al. 1998). However, G29-38 was nearly monoperiodic during the 1988 Whole Earth Telescope (WET) campaign (Winget et al. 1990). PG1351+489 is dominated by a single mode and its harmonics, and was WET target in 1995 (Alves et al. 2003). Using folded light curves, Montgomery (2005) obtained results for these two stars which are consistent with mixing length theory (MLT) and other calculations of convective transport.

This significant theoretical advance offers the first empirical determination of convection parameters in stars other than the Sun. However, a result from one star in each white dwarf instability strip provides an insufficient basis for global statements about the nature of the convection zones for all white dwarfs. The next logical step is to map a population spanning a range of temperatures and masses across each instability strip, enabling us to determine the depth of their convection zones as a function of $T_{\text {eff }}$ and $\log g$.

PG1351+489 and G29-38 are examples of relatively rare, large amplitude, monoperiodic pulsators where it is possible to fold long light curves to obtain a high signal-to-noise pulse shapes. Simulations by Montgomery (2006) show that this approach is not sufficient for the more common pulsators demonstrating variable complexity in their pulsation spectra. In these cases, we require at least $5 \mathrm{hr}$ of very high signal-tonoise photometry $(\mathrm{S} / \mathrm{N} \approx 1000)$ coupled with accurate real time frequency, amplitude, and phase information for the pulsations present in the high $\mathrm{S} / \mathrm{N}$ light curve. The frequency, amplitude, and phase information, provided by a long timebase, multisite observing run, is used to fit the high $\mathrm{S} / \mathrm{N}$ light curve and extract the convection parameters. The criteria for such a candidate star include nonlinear pulsations, a fairly bright target, and prior knowledge of the $l$ and $m$ values of the pulsations. The bright $\left(m_{\mathrm{v}}=13.5\right)$ DB GD358 fits these criteria. GD358 is the best studied DB pulsator, and the only DB with existing, detailed asteroseismology (Winget et al. 1994, hereafter W94; Bradley \& Winget 1994; Kepler et al. 2003, hereafter K03; Metcalfe et al. 2003).

In cooperation with the Delaware Asteroseismic Research Center (Provencal et al. 2005), we organized a WET run in May of 2006 (XCOV25) with GD358 as the prime target for Montgomery's light curve fitting technique. Our purpose was twofold: (1) obtain at least $5 \mathrm{hr}$ of $\mathrm{S} / \mathrm{N} \approx 1000$ photometry and (2) accurately identify the frequencies, amplitudes and phases present in GD358's current pulsation spectrum. The 2006 XCOV25 data set contains $\approx 436 \mathrm{hr}$ of observations, with $\approx 29 \mathrm{hr}$ of high $\mathrm{S} / \mathrm{N}$ photometry. While GD358 is the best studied DB pulsator, the star's behavior is by no means well understood. The 2006 data set contains a great deal of interesting information on GD358's pulsational behavior. Before we can proceed with detailed lightcurve fitting, we must thoroughly understand the data set, examine GD358's pulsation spectrum, and extract accurate frequency, amplitude, and phase information. In the following, we will present an overview of the data set and reduction procedures. Our discussion will expand the existing asteroseismological analysis of GD358, exploring the 2006 XCOV25 identified modes, combination frequencies, and multiplet structure. We will compare our results with previous observations and examine the complexity and evolution of GD358's pulsations over time. Our investigation will lead us to consider connections between GD358's pulsations, its convection zone, and a possible surface magnetic field. The remarkable event that occurred in 1996 August (K03) will play an important role in this discussion. Finally, we present implications for our understanding of GD358's physical properties and suggest future investigations.

\section{OBSERVATIONS AND DATA REDUCTION}

GD358 (V777 Her), the brightest $\left(m_{\mathrm{v}}=13.7\right)$ and best studied helium atmosphere white dwarf pulsator, was the target of the 25th Whole Earth Telescope (WET) run (XCOV25), the first sponsored by the Delaware Asteroseismic Research Center (DARC). The observations span 2006 May 12 to June 16, with the densest coverage between May 19 and May 31. Nineteen observatories distributed around the globe contributed a total of 88 individual runs (Table 1). The final XCOV25 light curve contains $436.1 \mathrm{hr}$ of high speed photometry.

A goal of any WET run is to minimize data artifacts by obtaining as uniform a data set as possible (Nather et al. 1990). Early WET runs (e.g., Winget et al. 1991) comprised mainly 3-channel blue-sensitive photomultiplier tube (PMT) photometers that monitored the target variable, a comparison star, and sky brightness. CCDs now bring improved sensitivity and better sky measurements, but individual CCDs have distinct effective bandpasses resulting in different measured amplitudes from different observing sites. Recent WET runs (examples include Kanaan et al. 2005 and Vuckovic et al. 2006) comprise a mixture of CCD and PMT observations, and XCOV25 is no exception. CCDs were employed at sixteen observatories, and 3-channel PMT photometers at the remaining three sites. We attempt to minimize bandpass issues by using CCDs with similar detectors 
Table 1

Journal of 2006 XCOV25 Observations

\begin{tabular}{|c|c|c|c|c|}
\hline Run Name & Telescope & Instrument & Date & $\begin{array}{c}\text { Length } \\
\text { (hr) }\end{array}$ \\
\hline konk20060512 & Konkoly 1.0 m & $\mathrm{CCD}$ & 2006 May 12 & 6.8 \\
\hline konk20060515 & Konkoly 1.0 m & $\mathrm{CCD}$ & 2006 May 15 & 6.3 \\
\hline mole20060515 & Moletai $1.65 \mathrm{~m}$ & PMT & 2006 May 15 & 1.3 \\
\hline mole20060517 & Moletai $1.65 \mathrm{~m}$ & PMT & 2006 May 17 & 3.5 \\
\hline konk20060517 & Konkoly 1.0 m & $\mathrm{CCD}$ & 2006 May 17 & 5.3 \\
\hline cuba20060517 & Mt. Cuba $0.6 \mathrm{~m}$ & $\mathrm{CCD}$ & 2006 May 18 & 5.4 \\
\hline kpno20060518 & KPNO $2.1 \mathrm{~m}$ & $\mathrm{CCD}$ & 2006 May 18 & 7.0 \\
\hline ctio20060518 & CTIO $0.9 \mathrm{~m}$ & $\mathrm{CCD}$ & 2006 May 19 & 4.4 \\
\hline kpno20060519 & KPNO $2.1 \mathrm{~m}$ & $\mathrm{CCD}$ & 2006 May 19 & 7.3 \\
\hline hawa20060518 & Hawaii $0.6 \mathrm{~m}$ & $\mathrm{CCD}$ & 2006 May 19 & 2.2 \\
\hline $\operatorname{luln} 20060519$ & Lulin $1.0 \mathrm{~m}$ & $\mathrm{CCD}$ & 2006 May 19 & 5.0 \\
\hline cuba20060519 & Mt. Cuba $0.6 \mathrm{~m}$ & $\mathrm{CCD}$ & 2006 May 20 & 2.5 \\
\hline ctio20060519 & CTIO $0.9 \mathrm{~m}$ & $\mathrm{CCD}$ & 2006 May 20 & 2.1 \\
\hline kpno20060520 & KPNO $2.1 \mathrm{~m}$ & $\mathrm{CCD}$ & 2006 May 20 & 7.6 \\
\hline hawa20060519 & Hawaii $0.6 \mathrm{~m}$ & CCD & 2006 May 20 & 1.7 \\
\hline ters 20060520 & Peak Terskol $2.0 \mathrm{~m}$ & $\mathrm{CCD}$ & 2006 May 20 & 5.8 \\
\hline cuba20060520 & Mt. Cuba $0.6 \mathrm{~m}$ & $\mathrm{CCD}$ & 2006 May 21 & 7.1 \\
\hline kpno20060521 & KPNO $2.1 \mathrm{~m}$ & $\mathrm{CCD}$ & 2006 May 21 & 7.3 \\
\hline ctio20060520 & CTIO $0.9 \mathrm{~m}$ & $\mathrm{CCD}$ & 2006 May 21 & 0.7 \\
\hline hawa20060520 & Hawaii $0.6 \mathrm{~m}$ & $\mathrm{CCD}$ & 2006 May 21 & 3.5 \\
\hline ters 20060521 & Peak Terskol $2.0 \mathrm{~m}$ & CCD & 2006 May 21 & 6.0 \\
\hline tueb20060521 & Tuebingen $0.8 \mathrm{~m}$ & $\mathrm{CCD}$ & 2006 May 21 & 6.6 \\
\hline lapa20060521 & La Palma WHT $4.2 \mathrm{~m}$ & $\mathrm{CCD}$ & 2006 May 22 & 0.9 \\
\hline cuba20060521 & Mt. Cuba 0.6 m & $\mathrm{CCD}$ & 2006 May 22 & 4.4 \\
\hline ctio20060521 & CTIO $0.9 \mathrm{~m}$ & $\mathrm{CCD}$ & 2006 May 22 & 4.5 \\
\hline kpno20060522 & KPNO $2.1 \mathrm{~m}$ & $\mathrm{CCD}$ & 2006 May 22 & 1.0 \\
\hline cuba20060521 & Mt. Cuba $0.6 \mathrm{~m}$ & $\mathrm{CCD}$ & 2006 May 22 & 1.7 \\
\hline hawa20060521 & Hawaii $0.6 \mathrm{~m}$ & $\mathrm{CCD}$ & 2006 May 22 & 7.8 \\
\hline $\operatorname{luln} 20060522$ & Lulin $1.0 \mathrm{~m}$ & $\mathrm{CCD}$ & 2006 May 22 & 4.5 \\
\hline konk20060522 & Konkoly 1.0 m & CCD & 2006 May 22 & 4.2 \\
\hline ters 20060522 & Peak Terskol $2.0 \mathrm{~m}$ & $\mathrm{CCD}$ & 2006 May 22 & 1.3 \\
\hline mcdo20060523 & McDonald $2.1 \mathrm{~m}$ & $\mathrm{CCD}$ & 2006 May 23 & 7.4 \\
\hline kpno20060523 & KPNO $2.1 \mathrm{~m}$ & $\mathrm{CCD}$ & 2006 May 23 & 2.9 \\
\hline hawa20060522 & Hawaii $0.6 \mathrm{~m}$ & $\mathrm{CCD}$ & 2006 May 23 & 6.6 \\
\hline boao 20060523 & BOAO $1.8 \mathrm{~m}$ & $\mathrm{CCD}$ & 2006 May 23 & 7.1 \\
\hline mcdo20060524 & McDonald $2.1 \mathrm{~m}$ & $\mathrm{CCD}$ & 2006 May 24 & 7.2 \\
\hline cuba20060523 & Mt. Cuba $0.6 \mathrm{~m}$ & $\mathrm{CCD}$ & 2006 May 24 & 4.0 \\
\hline hawa20060523 & Hawaii 0.6 m & $\mathrm{CCD}$ & 2006 May 24 & 3.7 \\
\hline boao20060524 & BOAO $1.8 \mathrm{~m}$ & $\mathrm{CCD}$ & 2006 May 24 & 6.7 \\
\hline ters20060524 & Peak Terskol $2.0 \mathrm{~m}$ & $\mathrm{CCD}$ & 2006 May 24 & 6.1 \\
\hline haut20060524 & OHP $1.93 \mathrm{~m}$ & PMT & 2006 May 24 & 2.3 \\
\hline mcdo20060525 & McDonald $2.1 \mathrm{~m}$ & $\mathrm{CCD}$ & 2006 May 25 & 6.4 \\
\hline hawa20060524 & Hawaii $0.6 \mathrm{~m}$ & $\mathrm{CCD}$ & 2006 May 25 & 5.7 \\
\hline ters 20060525 & Peak Terskol $2.0 \mathrm{~m}$ & $\mathrm{CCD}$ & 2006 May 25 & 7.1 \\
\hline haut 20060525 & OHP $1.93 \mathrm{~m}$ & PMT & 2006 May 25 & 4.2 \\
\hline ctio20060525 & CTIO $0.9 \mathrm{~m}$ & $\mathrm{CCD}$ & 2006 May 26 & 4.0 \\
\hline hawa20060525 & Hawaii $0.6 \mathrm{~m}$ & $\mathrm{CCD}$ & 2006 May 26 & 8.8 \\
\hline haut20060526 & OHP $1.93 \mathrm{~m}$ & PMT & 2006 May 26 & 4.0 \\
\hline ters 20060526 & Peak Terskol $2.0 \mathrm{~m}$ & $\mathrm{CCD}$ & 2006 May 27 & 7.1 \\
\hline ctio20060526 & CTIO $0.9 \mathrm{~m}$ & $\mathrm{CCD}$ & 2006 May 27 & 4.3 \\
\hline hawa20060526 & Hawaii $0.6 \mathrm{~m}$ & $\mathrm{CCD}$ & 2006 May 27 & 9.1 \\
\hline chin 20060527 & BAO $2.16 \mathrm{~m}$ & PMT & 2006 May 27 & 4.5 \\
\hline haut20060527 & OHP $1.93 \mathrm{~m}$ & PMT & 2006 May 27 & 4.6 \\
\hline cuba20060527 & Mt. Cuba $0.6 \mathrm{~m}$ & $\mathrm{CCD}$ & 2006 May 28 & 6.2 \\
\hline $\operatorname{lna} 20060528$ & Itajuba $1.6 \mathrm{~m}$ & $\mathrm{CCD}$ & 2006 May 28 & 3.1 \\
\hline mcdo20060528 & McDonald $2.1 \mathrm{~m}$ & $\mathrm{CCD}$ & 2006 May 28 & 0.7 \\
\hline mcdo20060528b & McDonald $2.1 \mathrm{~m}$ & $\mathrm{CCD}$ & 2006 May 28 & 7.2 \\
\hline hawa20060527 & Hawaii $0.6 \mathrm{~m}$ & $\mathrm{CCD}$ & 2006 May 28 & 7.8 \\
\hline chin 20060528 & BAO $2.16 \mathrm{~m}$ & PMT & 2006 May 28 & 5.2 \\
\hline haut 20060528 & OHP $1.93 \mathrm{~m}$ & PMT & 2006 May 28 & 5.2 \\
\hline vien20060528 & Vienna $1.0 \mathrm{~m}$ & $\mathrm{CCD}$ & 2006 May 28 & 2.2 \\
\hline cuba 20060528 & Mt. Cuba $0.6 \mathrm{~m}$ & $\mathrm{CCD}$ & 2006 May 29 & 3.4 \\
\hline mcdo20060529 & McDonald $2.1 \mathrm{~m}$ & $\mathrm{CCD}$ & 2006 May 29 & 8.2 \\
\hline cuba 20060528 & Mt. Cuba $0.6 \mathrm{~m}$ & $\mathrm{CCD}$ & 2006 May 29 & 2.6 \\
\hline hawa20060528 & Hawaii $0.6 \mathrm{~m}$ & $\mathrm{CCD}$ & 2006 May 29 & 9.1 \\
\hline
\end{tabular}

Table 1

(Continued)

\begin{tabular}{|c|c|c|c|c|}
\hline Run Name & Telescope & Instrument & Date & $\begin{array}{c}\text { Length } \\
\text { (hr) }\end{array}$ \\
\hline ters 20060529 & Peak Terskol $2.0 \mathrm{~m}$ & $\mathrm{CCD}$ & 2006 May 29 & 6.0 \\
\hline haut20060529 & OHP $1.93 \mathrm{~m}$ & PMT & 2006 May 29 & 5.3 \\
\hline cuba20060529 & Mt. Cuba $0.6 \mathrm{~m}$ & $\mathrm{CCD}$ & 2006 May 30 & 2.7 \\
\hline ters 20060530 & Peak Terskol $2.0 \mathrm{~m}$ & $\mathrm{CCD}$ & 2006 May 30 & 6.9 \\
\hline haut20060530 & OHP $1.93 \mathrm{~m}$ & PMT & 2006 May 30 & 4.2 \\
\hline hawa20060530 & Hawaii $0.6 \mathrm{~m}$ & CCD & 2006 May 31 & 8.7 \\
\hline chin 20060531 & BAO $2.16 \mathrm{~m}$ & PMT & 2006 May 31 & 3.9 \\
\hline ters 20060531 & Peak Terskol $2.0 \mathrm{~m}$ & $\mathrm{CCD}$ & 2006 May 31 & 7.0 \\
\hline nord20060606 & NOT $2.7 \mathrm{~m}$ & CCD & 2006 Jun 6 & 5.9 \\
\hline tueb20060607 & Tuebingen $0.8 \mathrm{~m}$ & $\mathrm{CCD}$ & 2006 Jun 7 & 4.2 \\
\hline nord20060607 & NOT $2.7 \mathrm{~m}$ & $\mathrm{CCD}$ & 2006 Jun 7 & 7.1 \\
\hline tueb20060608 & Tuebingen $0.8 \mathrm{~m}$ & $\mathrm{CCD}$ & 2006 Jun 8 & 4.7 \\
\hline nord20060608 & NOT $2.7 \mathrm{~m}$ & CCD & 2006 Jun 8 & 8.0 \\
\hline tueb20060609 & Tuebingen $0.8 \mathrm{~m}$ & $\mathrm{CCD}$ & 2006 Jun 9 & 5.4 \\
\hline nord20060609 & NOT $2.7 \mathrm{~m}$ & CCD & 2006 Jun 9 & 7.9 \\
\hline tueb20060610 & Tuebingen $0.8 \mathrm{~m}$ & CCD & 2006 Jun 10 & 5.2 \\
\hline tueb20060611 & Tuebingen $0.8 \mathrm{~m}$ & $\mathrm{CCD}$ & 2006 Jun 11 & 5.1 \\
\hline tueb20060612 & Tuebingen $0.8 \mathrm{~m}$ & $\mathrm{CCD}$ & 2006 Jun 12 & 4.5 \\
\hline lapa20060613 & La Palma WHT $4.2 \mathrm{~m}$ & CCD & 2006 Jun 13 & 1.8 \\
\hline tueb20060613 & Tuebingen $0.8 \mathrm{~m}$ & $\mathrm{CCD}$ & 2006 Jun 13 & 5.2 \\
\hline lapa20060614 & La Palma WHT $4.2 \mathrm{~m}$ & CCD & 2006 Jun 14 & 1.4 \\
\hline lapa20060615 & La Palma WHT $4.2 \mathrm{~m}$ & CCD & 2006 Jun 15 & 2.6 \\
\hline lapa20060616 & La Palma WHT $4.2 \mathrm{~m}$ & $\mathrm{CCD}$ & 2006 Jun 16 & 2.0 \\
\hline
\end{tabular}

and equipping each CCD with a BG40 or S8612 filter to normalize wavelength response and reduce extinction effects. The bi-alkali photomultiplier tubes are blue sensitive, so no filters are required. We also made every attempt to observe the same comparison star at each site, but given plate scale differences, that is not always possible.

We employ a $10 \mathrm{~s}$ contiguous integration time with the PMT photometers, while the cycle time for the CCD observations, including exposure and readout times, varies for each instrument. To illustrate the extremes, the Argos camera (Nather \& Mukadam 2004) at McDonald Observatory uses a frametransfer $\mathrm{CCD}$ and $5 \mathrm{~s}$ integrations with negligible deadtime, while the camera on the CTIO $0.9 \mathrm{~m}$ telescope operated by the SMARTS consortium uses $10 \mathrm{~s}$ integration and a $25 \mathrm{~s}$ readtime for a total cycle time of $\approx 35 \mathrm{~s}$.

Data reduction for the PMT observations follows the prescription outlined in Nather et al. (1990) and W94. In most cases, a third channel continuously monitored sky, allowing point by point sky subtraction. For two channel observations, the telescope is occasionally moved off the target/comparison for sky observations. We examined each light curve for photometric quality, and marked and discarded "bad" points. "Bad" points are those dominated by external effects such as cosmic rays or clouds. We divided GD358's light curve by the comparison star to remove first order extinction and transparency effects. If necessary, we fit a low order polynomial to the individual light curves to remove remaining low frequency variations arising from differential color extinction. We divided by the mean light curve value and subtracted 1 , resulting in a light curve with amplitude variations as fractional intensity (mmi). The unit is a linear representation of the fractional intensity of modulation $(1 \mathrm{mmi} \approx 1 \mathrm{mmag})$. We present our Fourier transforms $(\mathrm{FT})$ in units of modulation amplitude $\left(1 \mathrm{mma}=1 \times 10^{-3} \mathrm{ma}\right)$.

XCOV25 marked an evolution of WET headquarters' role in CCD data reduction. A standard procedure for a WET run is for observers to transfer observations to headquarters for analysis 


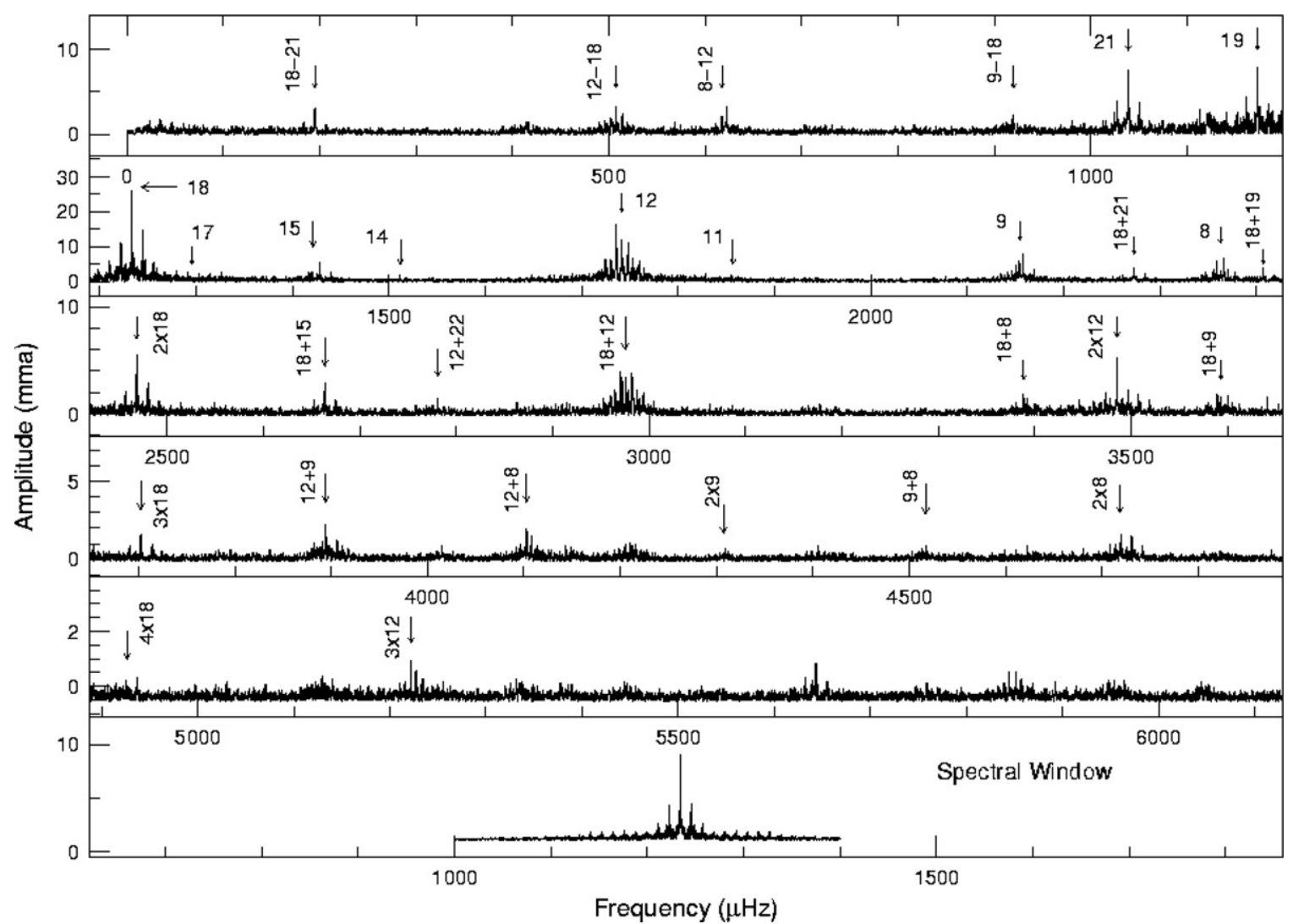

Figure 1. Fourier Transform of the 2006 GD358 photometry observations (note vertical scale in each panel). Arrows are labeled with $k$ values for independent modes (single values) and first order combination frequencies. The unlabeled peaks are second and third order combinations. The spectral window is plotted in the last panel. Table 2 lists exact identifications.

at the end of each night. In the past, CCD observers completed initial reductions (bias, flat, and dark removal) at their individual sites, performed preliminary aperture photometry, and transferred the result to WET headquarters. For XCOV25, the majority of participants transferred their raw images, enabling headquarters to funnel data through a uniform reduction pipeline. The few sites unable to transfer images nightly performed preliminary reductions on site using the same procedures as those at headquarters, and sent their images at a later date.

CCD data reduction follows the pipeline described by Kanaan et al. (2002). We corrected each image for bias and dark counts, and divided by the flat-field. Aperture photometry with a range of aperture sizes was performed on each image for the target and selected comparison stars. For each individual nightly run, we chose the combination of aperture size and comparison star producing the highest signal/noise as the final light curve. Each reduced CCD light curve consists of a list of times and fractional intensities. As with the photometer observations, we examined each light curve for photometric quality and discarded "bad" points.

Finally, we combined the individual PMT and CCD light curves to produce the complete light curve for XCOV25. This last step requires detailed assessment of overlapping observations. We make two assumptions in this process: (1) our observational technique is not sensitive to periods longer than a few hours, and (2) we assume GD358 oscillates about a mean light level. These assumptions allow us to carefully identify and correct vertical offsets in overlapping segments.

This data set does contain a significant fraction of overlapping lightcurves. We experimented with the effects of overlapping data on the FT by computing FTs with (1) all data included, (2) no overlapping data, where we kept those data with higher signal-to-noise ratio, and (3) weighting the overlapping light curves by telescope aperture size. We found no significant differences between the noise levels or FTs of overlapping versus non-overlapping versus weighted data.

Despite the favorable weather enjoyed by many participating sites, there are gaps within our light curve, especially near the beginning and end of XCOV25 when fewer telescopes were on-line. These gaps produce spectral leakage in the amplitude spectrum, resulting in a pattern of alias peaks associated with each physical mode that is not of astrophysical origin. To quantify this, we sampled a single sinusoid exactly as our original data and calculated its amplitude spectrum. The "spectral window" is the pattern produced by a single frequency in our data set. The Fourier transform and spectral window of the final complete light curve are given in Figure 1.

\section{FREQUENCY IDENTIFICATION}

\subsection{Stability}

Before we can look in detail at the 2006 XCOV25 FT, we must investigate GD358's amplitude and frequency stability over the entire timebase. GD358 is known for large scale changes in amplitudes and small but not insignificant frequency variations on a variety of timescales (K03). Amplitude and/or frequency variations produce artifacts in FTs, greatly complicating any analysis. We divided the data set into three chunks spanning $\approx 180 \mathrm{hr}$ and calculated the FT of each chunk (Figure 2). For 


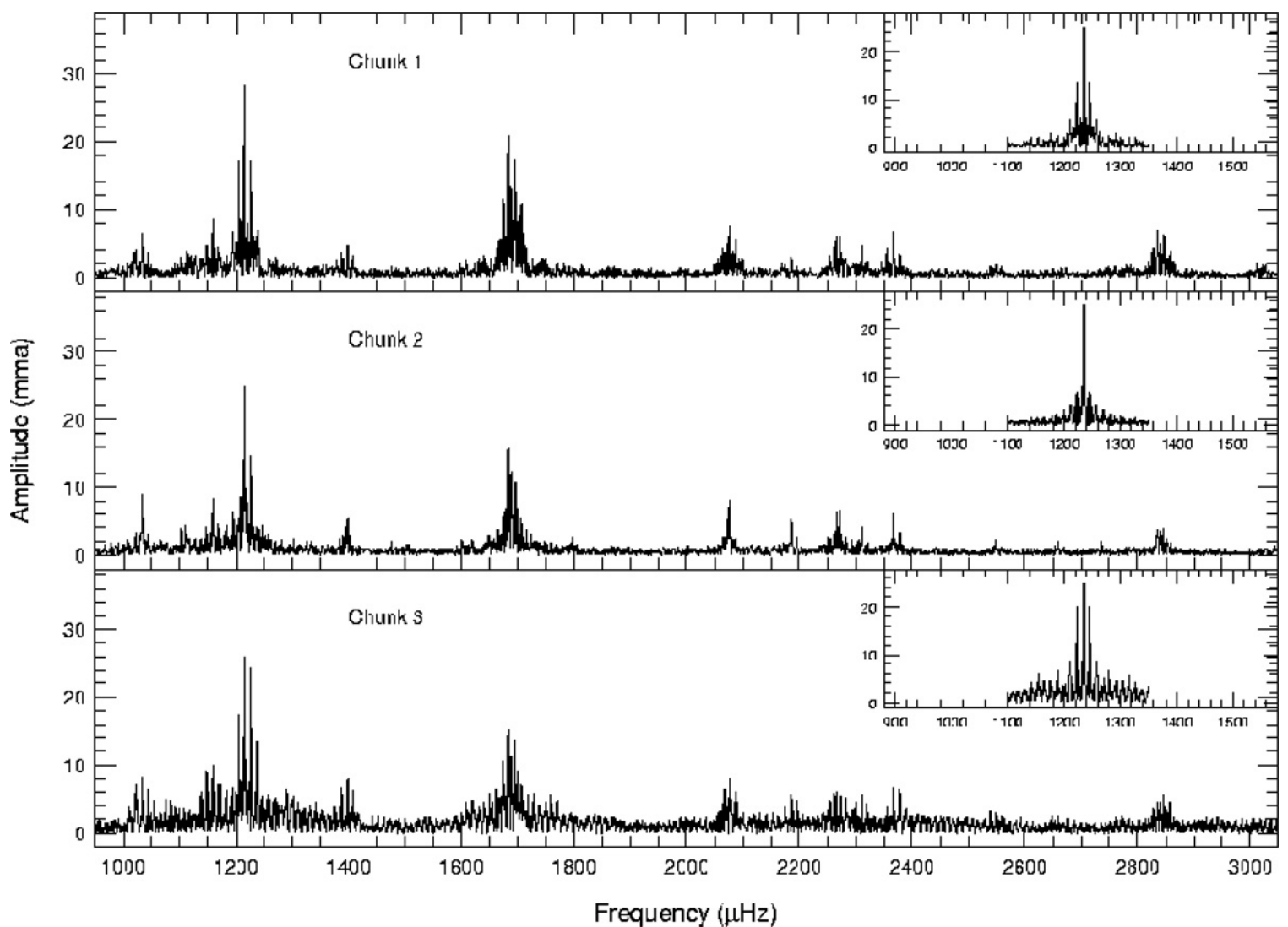

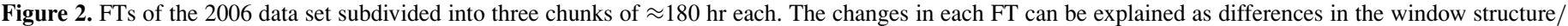
resolution for each chunk. GD358 was relatively stable over this time frame.

the dominant peak, the frequencies are consistent to within measurement error and the amplitudes remain stable to within $3 \sigma$. The differences between each FT are explained by variation in window structure and resolution from chunk to chunk. We are confident that GD358 was fairly stable over the length of $\mathrm{XCOV} 25$.

\subsection{The 2006 Fourier Transform}

To select the statistically significant peaks in the Fourier transform, we adopt the criterion that a peak have an amplitude at least 4.0 times greater than the average noise level in the given frequency range. This leads to a $99.9 \%$ probability that the peak is a real signal present in the data, and is not due to noise (Scargle 1982; Breger et al. 1993, K03, for example). Here, "noise" is defined as the average amplitude after prewhitening by the dominant frequencies in the FT, and is frequency dependent. This is a conservative estimate, as it is impossible to ensure that all of the "real" frequencies are removed when calculating the noise level. This is most certainly the case in GD358, where the FT contains myriad combination frequencies. Figure 3 displays the average amplitude, specified as the square root of the average power after prewhitening by 50 frequencies, as a function of frequency, for 2006, and previous WET runs on GD358 in 2000, 1994, and 1990.

The amplitude limit we select is very important in determining "real'signals. To confirm our uncertainty estimates, we performed several Monte Carlo simulations using the routine provided in Period04, software devoted to the statistical analysis of time series photometry (Lenz \& Breger 2005). The Monte Carlo routine generates a set of light curves using the original times, the fitted frequencies and amplitudes, and added
Gaussian noise. A least-squares fit is performed on each simulated light curve, with the distribution of fit parameters giving the uncertainties. Our Monte Carlo results are consistent with our average amplitude noise estimates (Table 2), confirming our use of the average amplitude after prewhitening.

For both Fourier analysis and multiple least squares fitting, we use the program Period04. The basic method involves identifying the largest amplitude resolved peak in the FT, subtracting that sinusoid from the original light curve, recomputing the FT, examining the residuals, and repeating the process. This technique is fraught with peril, especially in multiperiodic stars, where it is possible for overlapping spectral windows to conspire to produce alias amplitudes larger than real signals. Elimination of this alias issue was the driving motivation behind the development of the WET, whose goal is to obtain nearly continuous coverage over a long time baseline. Our current data set on GD 358 does contain gaps, but we have minimized the alias problem.

To illustrate the procedure we followed, let us examine the region of dominant power at $1234 \mu \mathrm{Hz}$ (Figure 4). Comparison of this region with the spectral window demonstrates that most of the signal is concentrated at $1234 \mu \mathrm{Hz}$. We fit the data with a sine wave to determine frequency, amplitude, and phase, and subtract the result from the original light curve. The second panel of Figure 4 shows the FT prewhitened by this frequency. Careful examination reveals two residual peaks (arrows) that are clearly not components of the spectral window. We next subtract a simultaneous fit of the $1234 \mu \mathrm{Hz}$ frequency and these two additional frequencies, with the results displayed in panel 3 of Figure 4. At this point, we must proceed with extreme caution. The remaining peaks, which correspond closely with aliases in the spectral window, are significant and cannot be ignored. 


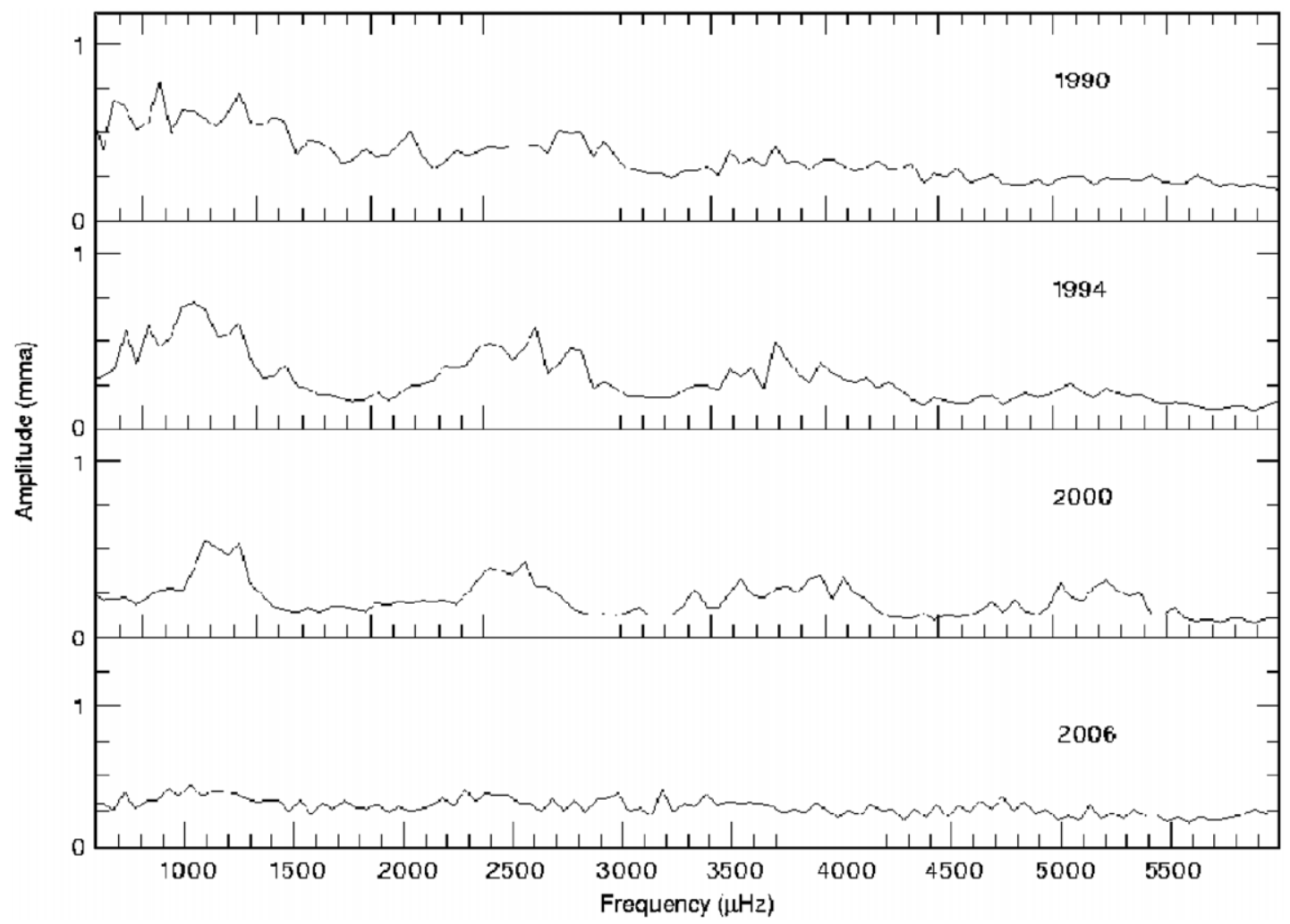

Figure 3. A comparison of the average noise from the 1990, 1994, 2000, and 2006 WET runs. Each data set was prewhitened by the 50 largest amplitude frequencies. This is a conservative estimate of the noise in each data set.

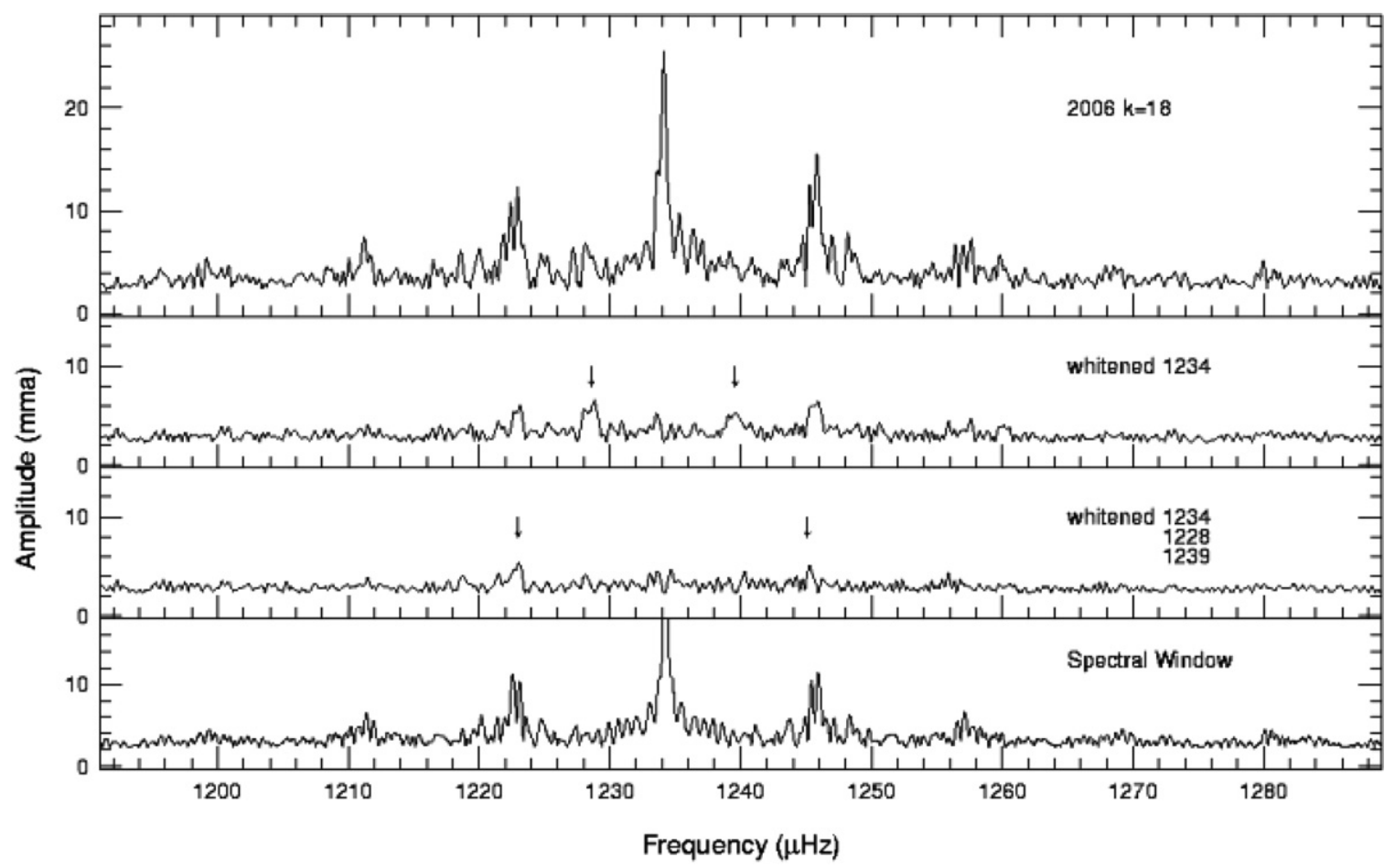

Figure 4. Demonstration of prewhitening using the dominant $1234 \mu \mathrm{Hz}$ mode in the $2006 \mathrm{FT}$. We begin with the removal of the largest amplitude resolved peak, a careful comparison of the residuals with the spectral window (last panel), and the subsequent removal of additional peaks.

We are faced with two possibilities: (1) these peaks represent real signals, and (2) amplitude modulation is present. While we have ruled out large modulations in frequency/amplitude during XCOV25, small scale amplitude modulation may be intrinsic to GD358, or artificially present in the data set. An examination of data from individual sites reveals that although the frequencies from each site are the same within the statistical uncertainties, two observatories, one using a CCD and one a 
Table 2

Table of 2006 Independent Frequencies

\begin{tabular}{|c|c|c|c|c|}
\hline$k(l=1)$ & $\begin{array}{c}2006 \text { Frequency } \\
(\mu \mathrm{Hz})\end{array}$ & $\begin{array}{l}\text { Amp } \\
(\mathrm{mma})\end{array}$ & $\mathrm{S} / \mathrm{N}$ & $\begin{array}{c}\mathrm{MC} \sigma_{\mathrm{amp}} \\
(\mathrm{mma})\end{array}$ \\
\hline 21 & $1039.076 \pm 0.002$ & $7.96 \pm 0.07$ & 27 & 0.08 \\
\hline 19 & $1173.042 \pm 0.002$ & $7.28 \pm 0.07$ & 25 & 0.08 \\
\hline 18 & $1222.751 \pm 0.001$ & $5.0 \pm 0.07$ & 18 & 0.6 \\
\hline 18 & $1228.792 \pm 0.002$ & $5.7 \pm 0.07$ & 19 & 0.07 \\
\hline 18 & $1234.124 \pm 0.001$ & $24 \pm 0.07$ & 88 & 0.09 \\
\hline 18 & $1239.540 \pm 0.002$ & $4.7 \pm 0.07$ & 18 & 0.07 \\
\hline 18 & $1245.220 \pm 0.003$ & $4.7 \pm 0.07$ & 17 & 0.6 \\
\hline 17 & $1295.533 \pm 0.008$ & $1.4 \pm 0.08$ & 5 & 0.6 \\
\hline 15 & $1421.012 \pm 0.008$ & $2.8 \pm 0.08$ & 7 & 0.07 \\
\hline 15 & $1423.942 \pm 0.008$ & $1.3 \pm 0.08$ & 6 & 0.07 \\
\hline 15 & $1429.210 \pm 0.002$ & $5.6 \pm 0.07$ & 23 & 0.07 \\
\hline 15 & $1434.784 \pm 0.008$ & $1.4 \pm 0.07$ & 4.4 & 0.06 \\
\hline 15 & $1440.622 \pm 0.008$ & $1.4 \pm 0.07$ & 4.3 & 0.06 \\
\hline 14 & $1512.010 \pm 0.007$ & $3.6 \pm 0.08$ & 8 & 0.07 \\
\hline 12 & $1736.302 \pm 0.001$ & $17.0 \pm 0.07$ & 75 & 0.07 \\
\hline 12 & $1737.962 \pm 0.007$ & $5.6 \pm 0.07$ & 8 & 0.08 \\
\hline 12 & $1741.665 \pm 0.001$ & $10.9 \pm 0.07$ & 50 & 0.07 \\
\hline 12 & $1743.738 \pm 0.002$ & $5.6 \pm 0.07$ & 8 & 0.07 \\
\hline 12 & $1746.673 \pm 0.007$ & $1.8 \pm 0.08$ & 8 & 0.08 \\
\hline 12 & $1749.083 \pm 0.001$ & $12.9 \pm 0.07$ & 50 & 0.07 \\
\hline 11 & $1856.845 \pm 0.009$ & $1.4 \pm 0.08$ & 6.4 & 0.08 \\
\hline $9^{-1}$ & $2150.395 \pm 0.003$ & $4.2 \pm 0.07$ & 17 & 0.07 \\
\hline $9^{0}$ & $2154.222 \pm 0.002$ & $5.5 \pm 0.07$ & 22 & 0.07 \\
\hline $9^{+1}$ & $2158.071 \pm 0.002$ & $7.2 \pm 0.07$ & 29 & 0.07 \\
\hline $8^{-1}$ & $2359.064 \pm 0.002$ & $5.87 \pm 0.07$ & 22 & 0.07 \\
\hline $8^{0}$ & $2363.058 \pm 0.007$ & $1.82 \pm 0.08$ & 6 & 0.07 \\
\hline $8^{+1}$ & $2366.523 \pm 0.002$ & $6.54 \pm 0.07$ & 23 & 0.07 \\
\hline
\end{tabular}

PMT, report consistently lower amplitudes, probably due to beating effects. Removing the suspect observations from the data set does not alter the prewhitening results for the $1234 \mu \mathrm{Hz}$ region. As an additional test, we closely examined the FTs from Figure 2. Prewhitening the $1234 \mu \mathrm{Hz}$ regions of each chunk consistently produces the same five peaks. Finally, we note that one of these peaks appears in combination with other modes (Table 3). Therefore, we believe our first possibility is most probable, all five peaks are real and are listed in Table 2.

We employed this procedure to identify frequencies satisfying our criteria of amplitudes $4 \sigma$ above the average noise. The large amplitude power at $1740 \mu \mathrm{Hz}$ is particularly complex. Unlike our example at $1234 \mu \mathrm{Hz}$, this mode contains three peaks with amplitudes over $10 \mathrm{mma}$ and contains as many as nine frequencies (Figure 5). Figure 6 demonstrates the prewhitening results for modes at 2154 and $2362 \mu \mathrm{Hz}$.

Our final identifications result from a simultaneous nonlinear least squares fit of 130 frequencies, amplitudes, and phases. We adopt the $l=1$ mode identifications for GD358 established in W94. We emphasize, however, that $k$, representing the number of nodes in the radial component of displacement from the center to the stellar surface, cannot be observationally determined and may not correspond precisely to the values given here. Tables 2 lists 27 identified independent frequencies. Table 3 presents significant combination frequencies. In the interest of space, we do not list all of the combination frequencies.
Table 3

2006 Combination Frequencies

\begin{tabular}{|c|c|c|c|c|c|}
\hline $\begin{array}{l}\text { Frequency } \\
(\mu \mathrm{Hz})\end{array}$ & $\begin{array}{c}\text { Combination(s) } \\
\mathrm{k}\end{array}$ & $\begin{array}{c}f_{\text {obs }}-f_{\text {calc }} \\
(\mu \mathrm{Hz})\end{array}$ & $\begin{array}{l}\text { Amp } \\
(\mathrm{mma})\end{array}$ & $(\mathrm{S} / \mathrm{N})$ & $R_{c}$ \\
\hline 195.085 & $1234-21$ & 0.04 & 2.7 & 7.7 & $\ldots$ \\
\hline 507.523 & $1741.7-1234$ & -0.02 & 3.0 & 8.2 & $\ldots$ \\
\hline 622.798 & $2358.1-1736.3$ & -1.0 & 2.8 & 7.5 & $\ldots$ \\
\hline 920.039 & $9^{0}-1234$ & -0.06 & 1.9 & 4.5 & $\ldots$ \\
\hline 2078.187 & $2 \times 1039.1$ & -0.1 & 0.74 & 3.2 & 2.4 \\
\hline 2273.244 & $1234+21$ & 0.05 & 4.2 & 10.8 & 9.6 \\
\hline 2407.205 & $1234+19$ & 0.07 & 3.8 & 9.7 & 9.1 \\
\hline 2462.989 & $1234+1228.8$ & 0.07 & 3.7 & 8.2 & 11.4 \\
\hline 2468.282 & $2 \times 1234$ & 0.034 & 5.1 & 13.1 & 1.8 \\
\hline 2479.358 & $1234+1245.2$ & 0.014 & 1.5 & 4.1 & 5.6 \\
\hline 2663.369 & $1234+1429.2$ & 0.04 & 2.9 & 7.3 & 9.4 \\
\hline 2780.786 & $1741.7+1039.1$ & 0.014 & 1.4 & 4.0 & 7.1 \\
\hline 2909.416 & $1736.3+19$ & 0.1 & 1.0 & 2.6 & 3.5 \\
\hline \multirow[t]{2}{*}{2964.917} & $1228.8+1736.3$ & -0.18 & 1.1 & 2.8 & 5.0 \\
\hline & $1222.8+1741.7$ & -0.46 & 1.1 & 2.8 & \\
\hline \multirow[t]{2}{*}{2970.400} & $1234+1736.3$ & 0.025 & 3.0 & 7.6 & 3.1 \\
\hline & $1228.8+1741.7$ & -0.057 & & & \\
\hline \multirow[t]{2}{*}{2972.085} & $1234+1737.9$ & 0.036 & 2.82 & 7.6 & 8.8 \\
\hline & $1222.8+1749.1$ & -0.22 & & & \\
\hline \multirow[t]{2}{*}{2975.814} & $1234+1741.7$ & -0.024 & 3.4 & 8.7 & 5.5 \\
\hline & $1239.5+1736.3$ & -0.039 & & & \\
\hline \multirow[t]{2}{*}{2977.885} & $1228.8+1749.1$ & 0.01 & 1.7 & 4.4 & 11.9 \\
\hline & $1234+1743.8$ & 0.02 & & & \\
\hline 2981.032 & $1239.5+1741.7$ & 0.17 & 1.3 & 4.0 & 11.2 \\
\hline 2981.947 & $1245.2+1736.3$ & -0.45 & 3.5 & 7.9 & 20.6 \\
\hline 2983.266 & $1234+1749.1$ & 0.057 & 3.1 & 7.9 & 4.66 \\
\hline 2988.643 & $1239.5+1749.1$ & -0.42 & 1.1 & 3.9 & 9.36 \\
\hline 3388.400 & $1234+9^{0}$ & 0.052 & 1.5 & 4.0 & 4.75 \\
\hline 3392.185 & $1234+2150.4$ & -0.013 & 1.7 & 4.4 & 7.35 \\
\hline 3472.552 & $2 \times 1736.3$ & -0.052 & 1.5 & 4.8 & 1.2 \\
\hline 3477.939 & $1736.3+1741.7$ & 0.06 & 1.7 & 4.5 & 4.0 \\
\hline \multirow[t]{2}{*}{3485.387} & $1741.7+1743.7$ & -0.02 & 5.7 & 16.7 & 11.6 \\
\hline & $1736.3+1749.1$ & 0.003 & 5.7 & 16.8 & \\
\hline 3490.720 & $1741.7+1749.1$ & 0.03 & 1.3 & 4.0 & \\
\hline 3593.136 & $1234+2359.1$ & -0.041 & 1.6 & 4.2 & 4.8 \\
\hline 3600.577 & $1234+2366.5$ & -0.071 & 1.7 & 4.5 & 4.5 \\
\hline 3702.443 & $3 \times 1234$ & 0.071 & 2.0 & 5.3 & \\
\hline 3890.607 & $1736.3+9^{0}$ & 0.08 & 1.5 & 4.8 & 7.0 \\
\hline 3894.392 & $1736.3+2158.1$ & 0.016 & 2.7 & 7.7 & 6.8 \\
\hline 3907.140 & $1749.083+2158.1$ & -0.014 & 1.6 & 5 & 7.7 \\
\hline 4095.326 & $1736.302+2359.1$ & -0.03 & 1.2 & 4.0 & 5.2 \\
\hline 4102.850 & $1736.302+2366.5$ & 0.024 & 2.2 & 7.3 & 4.0 \\
\hline 4108.130 & $1749.083+2359.1$ & -0.06 & 1.7 & 5.6 & 9.8 \\
\hline 4209.941 & $2 \times 1234+1741.7$ & 0.028 & 1.3 & 4.8 & \\
\hline 4308.536 & $2 \times 9^{0}$ & -0.092 & 1.0 & 4.6 & 11.7 \\
\hline \multirow[t]{3}{*}{4517.044} & $8^{0}+9^{0}$ & -0.24 & 1.1 & 4.7 & 48.5 \\
\hline & $2359.1+2158.1$ & 0.13 & & & \\
\hline & $2150.4+2366.5$ & -0.08 & & & \\
\hline 4719.483 & $2 \times 2359.064$ & -1.4 & 2.1 & 7.2 & 13.2 \\
\hline 4718.268 & $2 \times 2359.064$ & -0.14 & 1.1 & 4.7 & \\
\hline \multirow[t]{2}{*}{5221.734} & $1736.3+1741.7+1743.7$ & 0.034 & 1.3 & 4.2 & \\
\hline & $2 \times 1736.3+1749.1$ & -0.049 & & & \\
\hline 5643.452 & $1736.3+1749.1+2158.1$ & 0.05 & 1.2 & 4.1 & \\
\hline
\end{tabular}

\section{GD358 IN 2006}

\subsection{Independent Modes}

The 2006 XCOV25 data set illustrates GD358's continuing tendency for changing the distribution of amplitudes among its excited modes. Using the $k$ identifications established in W94, we detect power at $k=21,19,18,17,15,14,12,11,9$, and 8 . The principal frequency is at $1234 \mu \mathrm{Hz}(k=18)$ with an amplitude 


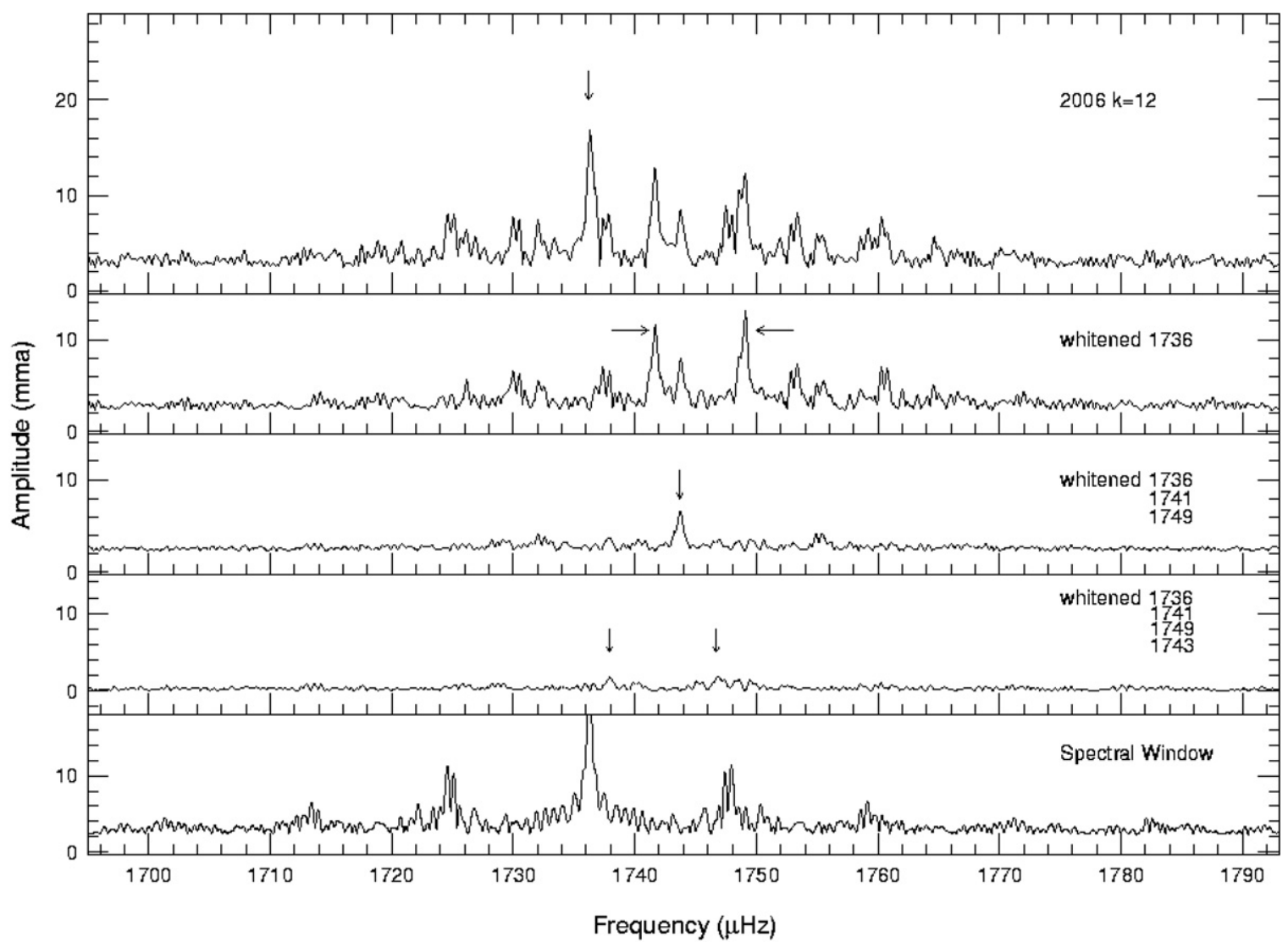

Figure 5. Prewhitening of the complex $k=12$ mode. We find three peaks over $10 \mathrm{mma}$ at 1736, 1741 and $1749 \mu \mathrm{Hz}$, and numerous additional peaks (Table 2). This mode could contain as many as 9 components.

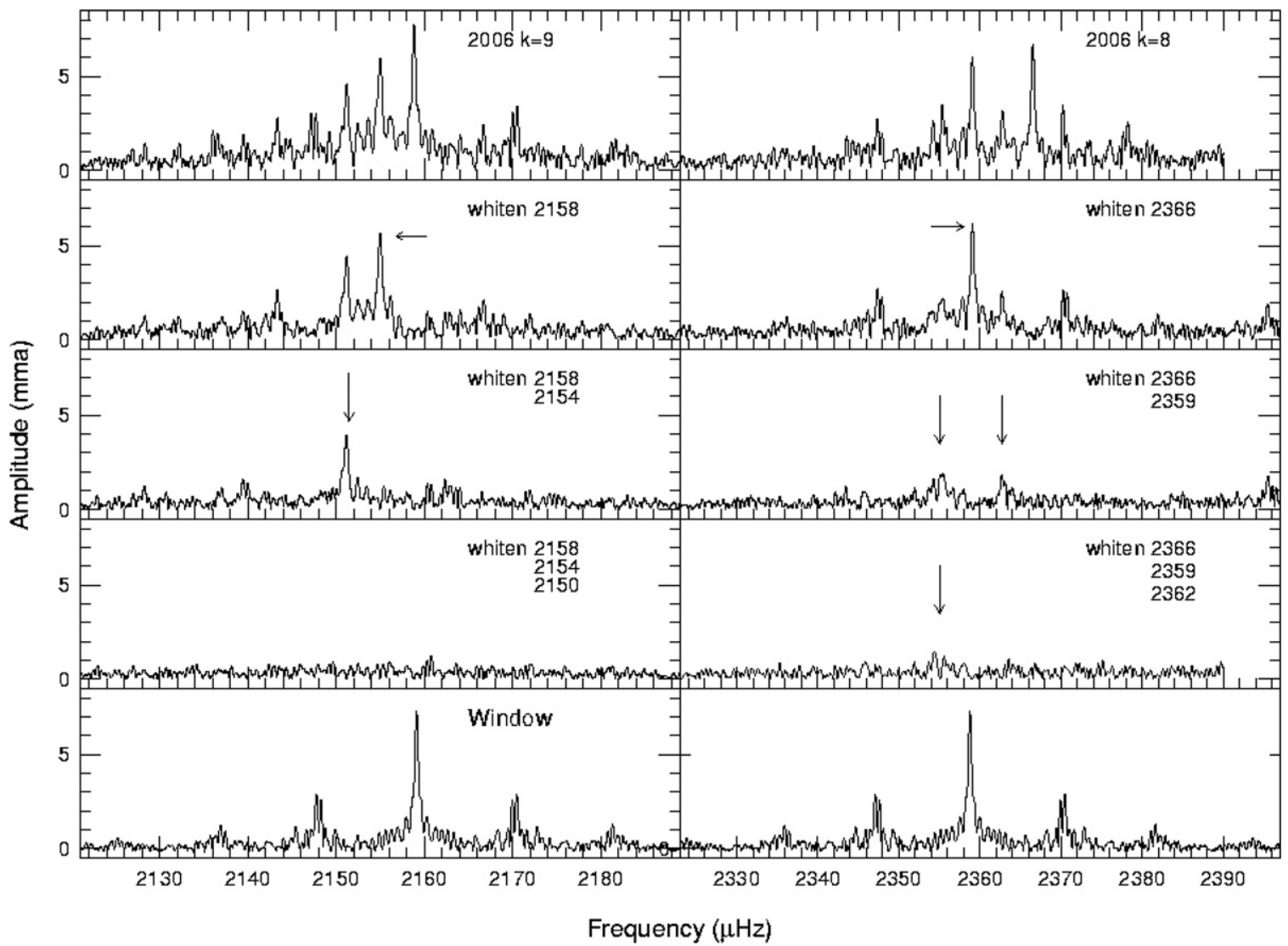

Figure 6. Prewhitening of the $k=9$ and 8 modes. These modes are triplets consistent with previous reports of W94 and K03. 


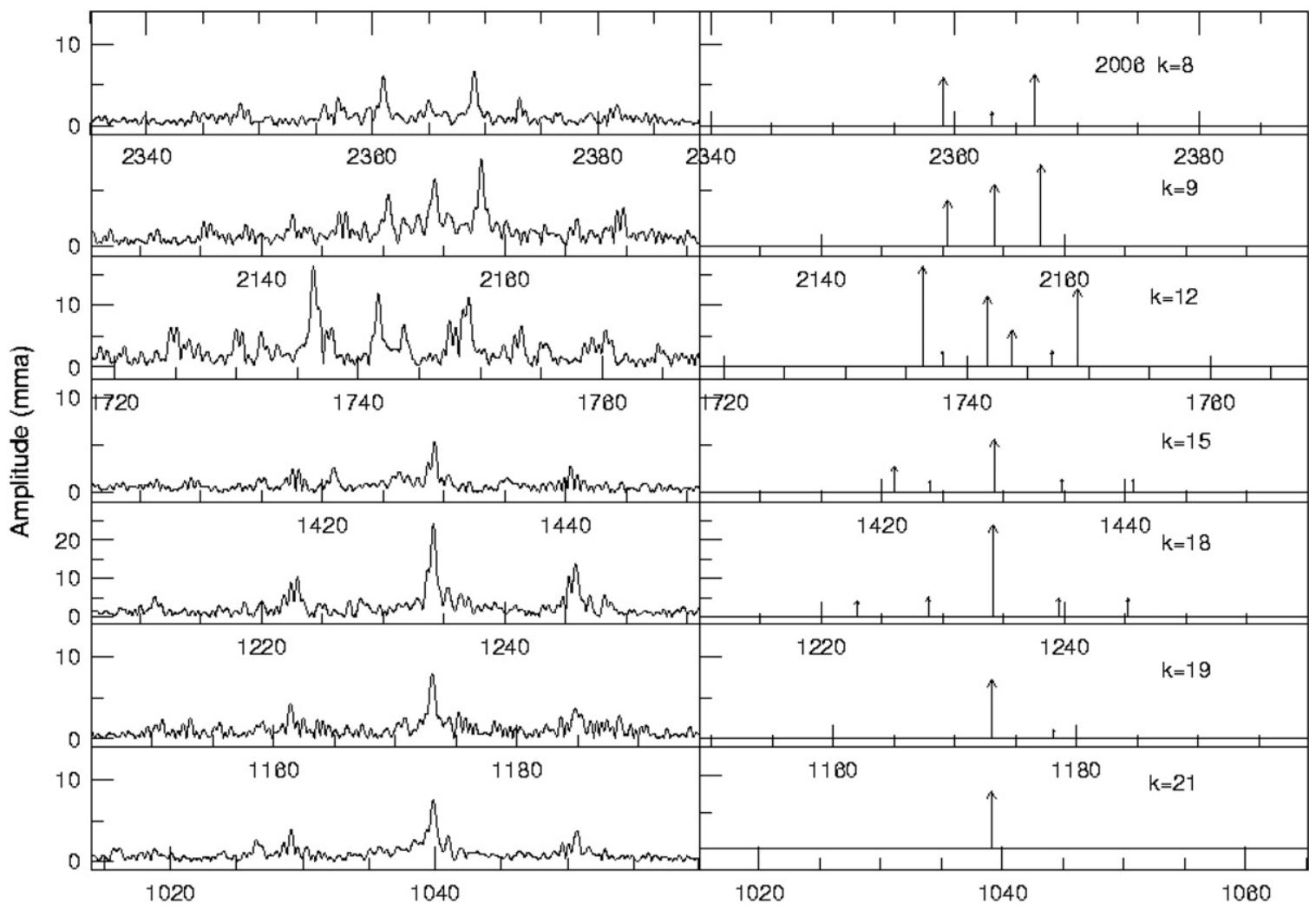

Frequency $(\mu \mathrm{Hz})$

Figure 7. A "snapshot" of the 2006 largest amplitude modes and their multiplet structure. The left panels plot the observed FT, and the right panels presents the prewhitening results. The $1 \sigma$ frequency errors are $\approx 0.001 \mu \mathrm{Hz}$. Each panel spans $50 \mu \mathrm{Hz}$. Note the changes in $y$ scale.

of 24 mma. The previously dominant $k=17$ and 15 modes (K03) are greatly diminished in amplitude (1.4 and $5.6 \mathrm{mma}$, respectively), and we do not detect the $k=16$ or $k=13$ modes reported by W 94 and K03. We cannot identify the suspected $l=$ 2 mode at $1255.4 \mu \mathrm{Hz}$ or $k=7$ at $2675.5 \mu \mathrm{Hz}$ noted by $\mathrm{K} 03$ ). K03 suggest that $k=7$ may have been excited to visibility via resonant coupling with $k=17$ and 16 . Since neither has significant amplitude in 2006 (Figure 1), it logically follows that this mode would not be detected. Perhaps the greatest surprise from XCOV25 is the appearance of prominent power at the predicted value for $k=12$, a region of the FT previously devoid of significant peaks.

Figure 7 presents a "snapshot" of multiplet structure in the XCOV25 FT. The only modes exhibiting structure with splittings in agreement with previous observations are $k=$ 9 and 8. We find average multiplet splittings of 3.83 and $3.75 \mu \mathrm{Hz}$, respectively. The dominant $k=18$ is a quintuplet with an average splitting of $5.6 \mu \mathrm{Hz}$. The $5.6 \mu \mathrm{Hz}$ splitting also appears in the $k=15$ and $k=12$ modes. The $k=15$ mode contains five peaks, only three of which are split by $5.6 \mu \mathrm{Hz}$. The $k=12$ mode could contain as many as nine components, if we relax our $4.0 \sigma$ detection criteria slightly. A possible interpretation for $k=12$ is two, perhaps three, overlapping triplets, each with $\approx 5.6 \mu \mathrm{Hz}$ splitting. An $l=2$ mode is predicted at $\approx 1745 \mu \mathrm{Hz}$, so a second possible interpretation is an overlap of $l=1$ and $l=2$ modes. We point out that the other high- $k$ modes $(17,16,14$, and 13$)$ reported by W94 to have frequency splittings of $\approx 6 \mu \mathrm{Hz}$ are either not detected here, or do not have sufficient amplitude to investigate multiplet structure.
Our goal for XCOV25 is the identification/confirmation of $l$ and $m$ values for modes in GD358's 2006 light curve. This information is required to fit our high signal-to-noise lightcurve using Montgomery's technique. However, the 2006 multiplet structure proves to be puzzling. In the limit of slow rotation, we expect each mode to be split into $2 l+1$ components. Except for $k=9$ and $k=8$, we do not find the distinct triplets attributable to $l=1$. Indeed, $k=18$ is a quintuplet at first glance suggesting $l=2$, and $k=15$ and 12 are both complex but lacking equal splittings. We cannot confirm the $l$ or $m$ designations of W94 and K03 based on multiplet structure alone. Fortunately, the identification of the spherical degree of GD358's pulsation modes as $l=1$ does not rely solely on multiplet structure. Table 4 presents the mean period spacing for XCOV25, as well as WET runs from 1990, 1991, 1994, and 2000. The average period spacing, which is dependent on stellar mass, is $38.6 \mathrm{~s}$. If GD358's modes are consecutive $l=1$, the period spacing corresponds to a stellar mass of $\approx 0.6 M_{o}$, in agreement with spectroscopic results (Beauchamp et al. 1999). If they are consecutive $l=2$ modes, the derived stellar mass becomes $\approx 0.2 M_{o}$, making GD358 one of the lowest mass field white dwarfs known and incompatible with the spectroscopic log $g$. In addition, the distance of $42 \pm 3 \mathrm{pc}$ derived from GD358 models assuming $l=1$ is consistent with the trigonometric distance of $36 \pm 4 \mathrm{pc}$. The distance derived derived from models assuming $l=2$ is $\approx 75$ pc (Bradley \& Winget 1994). Is it possible that we have an amalgam of $l=1$ and 2 modes? $l=2$ modes are predicted to be quintuplets, fitting with our results for $k=$ 18. However, we expect the ratio of rotational splittings between $l=1$ and $l=2$ to be $R_{1,2}=0.60$ (Winget et al. 1991). Assuming 
Table 4

Mean Period Spacing

\begin{tabular}{ccc}
\hline \hline Year & Time & $\Delta P_{\mathrm{av}}$ \\
& BJED & $(\mathrm{s})$ \\
\hline 1990 & 2440831.772 & $38.60 \pm 0.3$ \\
1991 & 2448356.716 & $38.39 \pm 0.3$ \\
1994 & 2449474.998 & $38.67 \pm 0.3$ \\
2000 & 2451702.402 & $38.77 \pm 0.3$ \\
2006 & 2453868.311 & $38.77 \pm 0.3$ \\
\hline
\end{tabular}

Notes. The spacing from the "period transform is given as PT, and the value from the subtracting of low- and high- $k$ periods is " $\mathrm{K} . "$

$k=8$ and 9 are $l=1$ as indicated by their triplet structure, we expect an $l=2$ splitting of $6.3 \mu \mathrm{Hz}$, much larger than the observed $5.6 \mu \mathrm{Hz}$ splitting. We have no easy explanation for the $5.6 \mu \mathrm{Hz}$ splitting, and hesitate to identify $k=18$ as an $l=2$ mode solely on the basis of a quintuplet. The $k=12$ and $k=15$ modes contain the mysterious $5.6 \mu \mathrm{Hz}$ splitting, but the splittings are unequally spaced. The "best fit" pulsation models do predict $l=2$ modes at $1250 \mu \mathrm{Hz}$ (near $k=18$ ) and $1745 \mu \mathrm{Hz}$ (near $k=12$ ) (Metcalfe et al. 2003). The $1250 \mu \mathrm{Hz}$ mode is not detected in this data set. Some of the complex peaks at $k=12$ could correspond to an underlying $l=2$ mode, but the splittings do not support this conclusion. Finally, optical and UV radial velocity variations have been used to determine the $l$ values for several modes in GD358, including $k=18$. Kotak et al. (2003) identified radial velocities corresponding to $k=$ $18,17,15,9$, and 8 in their time-resolved optical spectra. They determined that these modes all share the same $l$ value, which is probably $l=1$. Castanheira et al. (2005) use HST UV time resolved spectroscopy to determine that $k=9$ and 8 are best explained as $l=1$.

While we are fairly certain that the majority of GD358's modes are $l=1$, except for $k=9$ and 8 , we cannot directly provide $m$ identifications for the observed multiplet components or confirm the $m$ identifications of W94 and K03. For this data set, we must resort to indirect means. In the next section, we examine the combination frequencies, and, limiting ourselves to $l=1$, explore their potential for pinning down the multiplet $m$ components in GD358.

\subsection{Combination Frequencies}

GD358's 2006 FT contains a rich distribution of combination frequencies, from differences to simple harmonics to fourth order combinations. Combinations peaks are typically observed in the FTs of large amplitude pulsators (Dolez et al. 2006; Yeates et al. 2005; Thompson et al. 2003; Handler et al. 2002, W94). Their frequencies correspond to integer multiples of a single frequency (harmonics), combinations (both sums and differences) of different components of a given multiplet, or combinations (again both sums and differences) of components of different modes (cross combinations). The general consensus on the origin of these combinations dictates that they are not independent modes, but are the result of nonlinear mixing induced by the convection zone in the outer layers of the star (Brickhill 1992; Wu 2001; Ising \& Koester 2001). The convection zone varies its depth in response to the pulsations, distorting and delaying the original sinusoidal variations, and redistributing some power into combination frequencies. While combinations themselves do not provide additional direct information about the interior structure of the star, they are sensitive to mode geometry, making them potential tools for identifying $l$ and/or $m$ values and orientation for each mode.
$\mathrm{Wu}$ (2001) provides a theoretical overview of combinations and their interpretation. Handler et al. (2002) and Yeates et al. (2005) apply Wu's approach to several ZZ Ceti (DA) stars. Yeates et al. (2005) focus on the dimensionless ratio $R_{c}$ first introduced by van Kerkwijk et al. (2000). The theoretical value of $R_{c}$ is given as $R_{c}=F\left(\omega_{i}, \omega_{j}, \tau_{\mathrm{c}_{0}}, 2 \beta+\gamma\right) G(l, m, \theta)$. The first term, $F$, includes the frequencies of the parent modes $\left(\omega_{i}, \omega_{j}\right)$, as well as properties inherent to the star, such as the depth of the stellar convection zone at equilibrium $\left(\tau_{\mathrm{c}_{0}}\right)$ and the sensitivity of the convection zone to changes in temperature $(\beta, \gamma)$. The second term, $G$, includes geometric factors such as the $l$ and $m$ values of the parent modes, and the inclination angle of the pulsation axis. Both Wu (2001) and Yeates et al. (2005) present theoretical predictions for various combinations of $l$ and $m$ values.

The observed value of $R_{c}$ is defined as the ratio between the observed amplitude of a combination and the product of the observed amplitudes of its parent modes, including a correction factor incorporating the bolometric correction and an estimate for the convective exponent. The convective exponent appears in theoretical estimations of the thermal response timescale of the convection zone. From Montgomery (2008), we estimate the bolometric flux correction for GD358 to be 0.35 and the convective exponent to be 25 . For harmonic combinations, this produces a corrective factor of 0.22 . Cross combinations include an additional factor of 2 , resulting in a correction of 0.44 . With this formalism, and limiting ourselves to the previous identification of the principal modes as $l=1$, and with the smorgasbord of combination frequencies present in GD358, it should be possible to determine $m$ values of the parent modes, to provide limits on the inclination angle, and to further study convection in white dwarfs. However, interpretation of the combination frequencies is not as straightforward as we would hope.

The analysis of $R_{c}$ for harmonic combinations is simplified by the fact that we are certain that the combination contains a single parent, and so single values for $l$ and $m$. In the 2006 data set, we detect the dominant $1234 \mu \mathrm{Hz}$ mode's first, second, and third harmonics, and place upper amplitude limits of $\approx 0.3 \mathrm{mma}$ on higher orders. The first harmonic, at $2468.282 \mu \mathrm{Hz}$ with an amplitude of $5.1 \mathrm{mma}$, is the 12th highest amplitude peak in the FT and the second highest amplitude combination frequency observed.

The second largest mode and most complicated multiplet in the 2006 FT is $k=12$. Focusing on its three large amplitude components (Table 3), we find the first harmonic of $1736 \mu \mathrm{Hz}$, near $3472 \mu \mathrm{Hz}$ with an amplitude of $1.5 \mathrm{mma}$. Interestingly, the largest peak near $3472 \mu \mathrm{Hz}$ is actually a sum of $k=12$ components (see Table 3), not a simple harmonic, a behavior distinctly different from $k=18$. We place an upper limit of $0.5 \mathrm{mma}$ for a 1st harmonic of $1741.67 \mu \mathrm{Hz}, 0.6 \mathrm{mma}$ for $1749.08 \mu \mathrm{Hz}$, and upper limits of $0.3 \mathrm{mma}$ for all higher orders for all other components of $k=12$.

For the remaining modes in Table 2, we detect the first harmonic of $1039 \mu \mathrm{Hz}(k=21)$ with an amplitude of $0.7 \mathrm{mma}$. For $k=19,17,15,14$, and 11, we place upper limits of $0.5 \mathrm{mma}$ for their first harmonics. The $2154 \mu \mathrm{Hz}$ peak, identified as $k=9$, $m=0\left(k=9^{0}\right)$ in W94, has a first harmonic with an amplitude of $1.0 \mathrm{mma}$. It is surprising that we do not detect a harmonic of the larger amplitude $k=9^{+1}$ component. We place upper limits of $0.4 \mathrm{mma}$ for harmonics of the $k=9^{ \pm 1}$ components. Finally, the $2359 \mu \mathrm{Hz}$ component of $k=8$, identified as $k=8^{-1}$ in W94, has a first harmonic at $4719.483 \mu \mathrm{Hz}(2.1 \mathrm{mma})$. We place upper limits of $0.4 \mathrm{mma}$ for harmonics of the $k=8^{0}$ and 


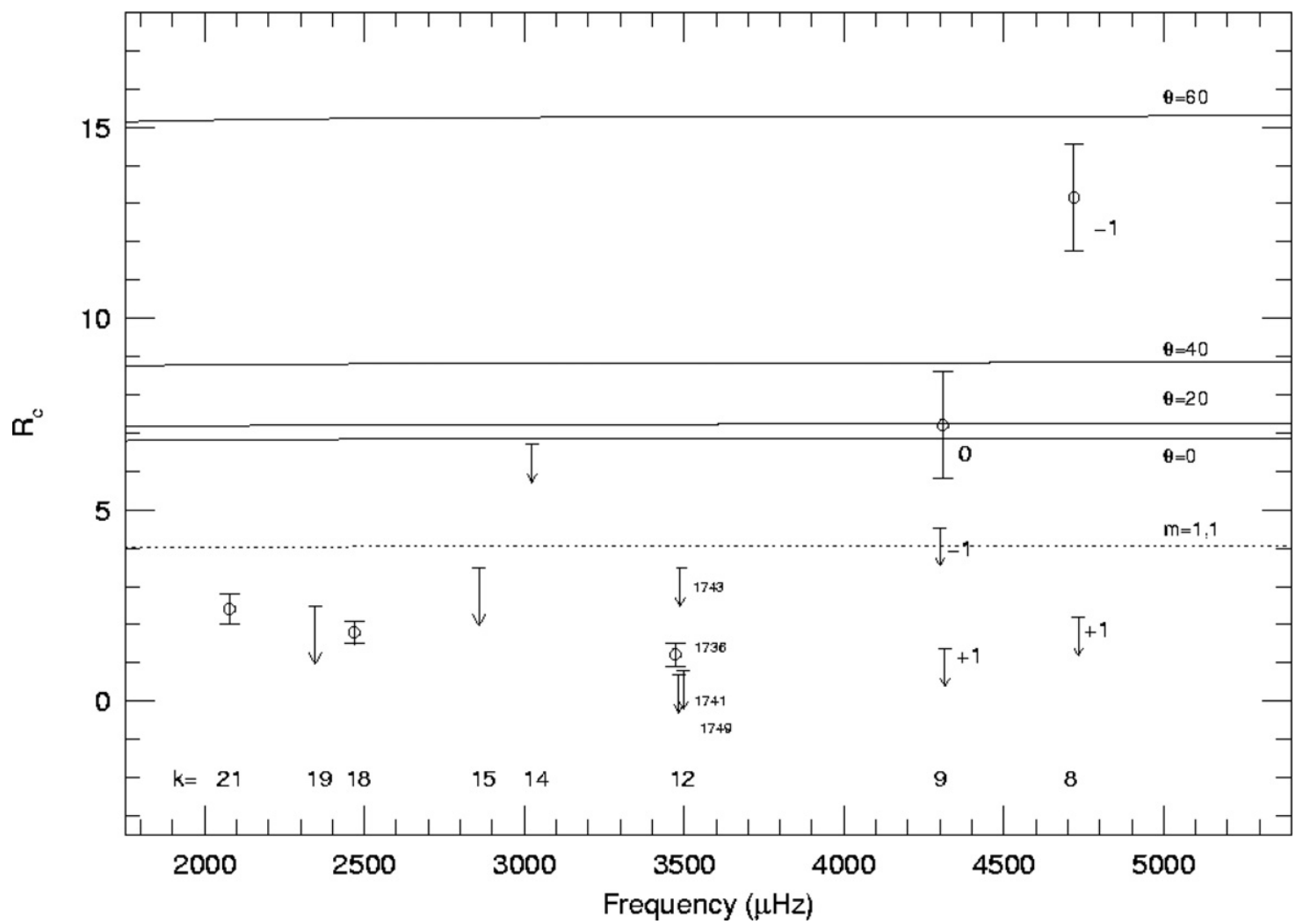

Figure 8. Observed $R_{c}$ values (ratio of amplitude of the combination to the product of the parent amplitudes) for detected harmonics. The open circles denote detected harmonics with estimated errors, and the vectors plot upper limits on other harmonics. The solid lines represent theoretical values for the harmonics of $m=0$ modes for inclinations of $0,20,40$, and $60^{\circ}$. The dotted line gives the theoretical value for the harmonics of $m=1$ and $m=-1$ modes, which do not depend on inclination. We rely on previous identifications of $m$ values for $k=9$ and 8

$k=8^{+1}$ components. We note again that the larger amplitude $k=8^{+1}$ does not have a significant harmonic.

We begin by plotting the observed values of $R_{c}$ for first order harmonics in Figure 8. If no harmonic is detected, we present upper limits. Since we do not have $m$ identifications for most modes but we know that $m$ must be the same for harmonic parents, we plot theoretical predictions for $l=1, m=0,0$ combinations for inclinations of $0,20,40$, and $60^{\circ}$ (solid lines) and $l=1, m=1,1$ and $m=-1,-1$ (dotted line). $R_{c}$ values for $m=1,1$ and $m=-1,-1$ combinations do not depend on inclination. Surprisingly, the observed $R_{c}$ values and upper limits for the high- $k$ modes are lower than the theoretical predictions. We could argue that, under the theoretical assumptions of $\mathrm{Wu}$ (2001), the detected high- $k$ multiplet components are not consistent with $m=0$.

From the $k=9$ harmonics, we find behavior closer to theoretical predictions. The $k=9^{0}$ harmonic is consistent with an inclination of between 0 and $40^{\circ}$, while the $k=9^{ \pm 1}$ components are in the direction of theoretical predictions. However, for $k=8$, the $k=8^{-1}$ harmonic has a larger value of $R_{c}$ than the $k=8^{+1}$ upper limit, something that is difficult to explain using current theory and simple viewing arguments. The upper limit of $R_{c}$ for $k=8^{0}$ is 29 , and is not shown in Figure 8.

The most eye-catching example of cross combination peaks is found near $3000 \mu \mathrm{Hz}$ (Figure 1), corresponding to linear combinations between the two largest multiplets, $k=18$ and $k=12$. Our resolution and sensitivity are sufficient to reveal nine combinations, with amplitudes ranging from $3.4 \mathrm{mma}$ to $1.1 \mathrm{mma}$. In several cases, multiple parent identifications are possible for each mode. For the following discussion, we include identifications for detected combinations involving at least one large amplitude parent, with most including the $1234 \mu \mathrm{Hz}$ mode in combination with components of $k=12$ (Table 3). The $1234 \mu \mathrm{Hz}$ mode also produces large amplitude combinations (4.2 and $3.8 \mathrm{mma})$ with the $1039(k=21)$ and $1173(k=19) \mu \mathrm{Hz}$ modes.

Figure 9 presents observed $R_{c}$ values and upper limits for first order cross combinations including the dominant $1234 \mu \mathrm{Hz}$ mode as one parent. We include the same theoretical predictions as in Figure 8, adding predictions for $l=1, m=+1,-1$ combinations for inclinations of 20,40 , and $60^{\circ}$ (dotted lines), and $m=0, \pm 1$ combinations (dot/dash line). The $R_{c}$ values for combinations of $1234 \mu \mathrm{Hz}$ with the high- $k$ modes $21,19,15$, and one component of $k=12(1739 \mu \mathrm{Hz})$ are consistent with either $m=0,0$ combinations with an inclination of roughly $40^{\circ}$ or $m=+1,-1$ combinations with an inclination closer to $50^{\circ}$. Recalling that the harmonic combinations (Figure 8) argue that none of the high- $k$ modes are $m=0$, the second choice of $m=+1,-1$ combinations seems more likely. This further implies that $1234 \mu \mathrm{Hz}$ is either $m=+1$ or $m=-1$, and the other modes are all the remaining value.

The relative values of $R_{c}$ among the $3000 \mu \mathrm{Hz}$ combinations $(k=18+12)$, while independent of most model parameters, do depend on inclination, and should reflect their respective projection in our line of sight. We should find $R_{c}$ values corresponding to combinations of $m=0, \pm 1, m=0,0$, $m=-1,+1$, and $m=$ same. However, even incorporating our tenuous identification of $1234 \mu \mathrm{Hz}$ as $m=-1$, given the number of multiplet components and large uncertainties in the inclination angle, our analysis based on $\mathrm{Wu}$ (2001) predictions produces no clear identification. 


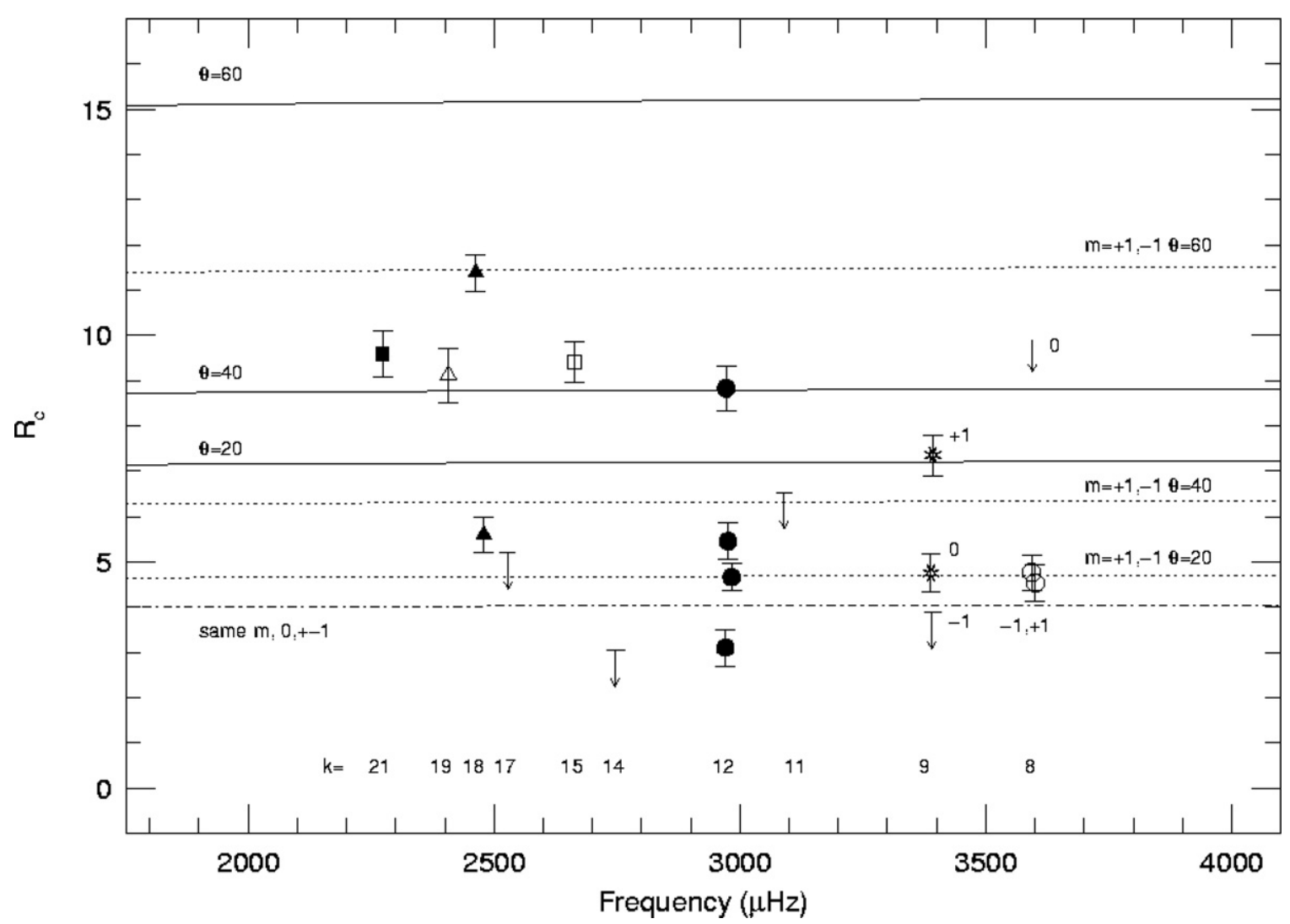

Figure 9. Observed $R_{c}$ values (ratio of amplitude of the combination to the product of the parent amplitudes) for cross combinations including $1234 \mu \mathrm{Hz}$ as one parent mode. The $k$ value of the second parent is given along the bottom of the figure, and different point types indicate combinations of $1234 \mu \mathrm{Hz}$ with different $k \mathrm{~s}$. The solid lines represent theoretical predictions for $m=0,0$ combinations with inclinations of 20,40 , and $60^{\circ}$, the dotted lines are for $m=+1,-1$ combinations with the same inclinations, and the dot/dash line represents $m=1,1$ and $m=-1,-1$, and $m=0, \pm 1$ combinations. Error bars are $1 \sigma$ formal errors.

Our analysis of GD358's combination frequencies is complex. We find a very tenuous identification for the $1234 \mu \mathrm{Hz}$ mode of $m= \pm 1$, requiring the remaining high- $k$ modes' largest component to be the remaining value. We find no clear $m$ identifications for the components of $k=12$. This mode is clearly more complex than the simple multiplet structure expected in the limit of slow rotation.

There are multiple reasons why GD358's combination frequencies defy simple interpretation. First, this star simply has too many principal modes simultaneously excited to large amplitude. The cross-talk between them is large, and the perturbative treatment of $\mathrm{Wu}$ (2001) may not be valid. Second, both $\mathrm{Wu}$ (2001) and Yeates et al. (2005) assume the intrinsic amplitudes are the same for every component in a multiplet, and this may simply not be the case for GD358. Third, the theoretical value of $R_{c}$ relies on correct identification of $l$ and $m$, although in simpler cases, as in Yeates et al. (2005), it can be used as a mode identification technique. While we are fairly certain that GD358's pulsations are $l=1$, the assumption of a classical triplet split by rotation is not valid for GD358's high- $k$ modes. Finally, we raise a possibility suggested by the $k=9$ and 8 components and their harmonics: to first order, the $m=0$ components sample the radial direction, and the $m= \pm 1$ components sample azimuthal direction. Components of the same mode have the same inclination, so cancellation effects should be the same. The $k=9$ and 8 multiplet amplitudes and presence/absence of harmonics argue that something is interrupting the azimuthal symmetry of GD358. It is possible that the pulsations do not all share the same inclination, in which case they would not combine as expected by $\mathrm{Wu}$ (2001). The oblique pulsator model is well known, especially in the case of roAp stars. For example, Bigot \& Dziembowski (2002) show that the main mode of HR 3831, a pulsating roAp star, departs from alignment with the magnetic axis. GD358's combinations are trying to tell us something important about the pulsation geometry, but we do not yet understand how to interpret it.

While the general frequencies of the high- $k$ multiplets agree with the identifications of W94 and K03, the 2006 multiplets themselves are complex, and cannot be explained by simply invoking rotation using standard theory and simple geometric viewing arguments. Except for the $k=9$ and $k=8$ modes, we do not find triplet structure as reported in W94 and cannot provide $m$ identifications. The facts that the multiplets do change and the 2006 combinations do not conform to theoretical predictions leads us to expand our investigation to include archival observations of GD358. In the following, we will examine the structure of GD358's multiplets in detail over timescales of years.

\section{MULTIPLET STRUCTURE CHANGE AND COMPLEXITY}

Each multiplet in GD358's FT should represent a quantized $g$-mode pulsation, described theoretically by a spherical harmonic of index $l$ and overtone $k$, which has been split into multiple components $(2 l+1)$ by the star's rotation. As component frequencies are determined by the star's structure and rotation rate, we would expect the multiplet structure to remain stable over long time periods. The classic example is the DO pulsator PG1159-035, which exhibits prototypical triplets, cor- 


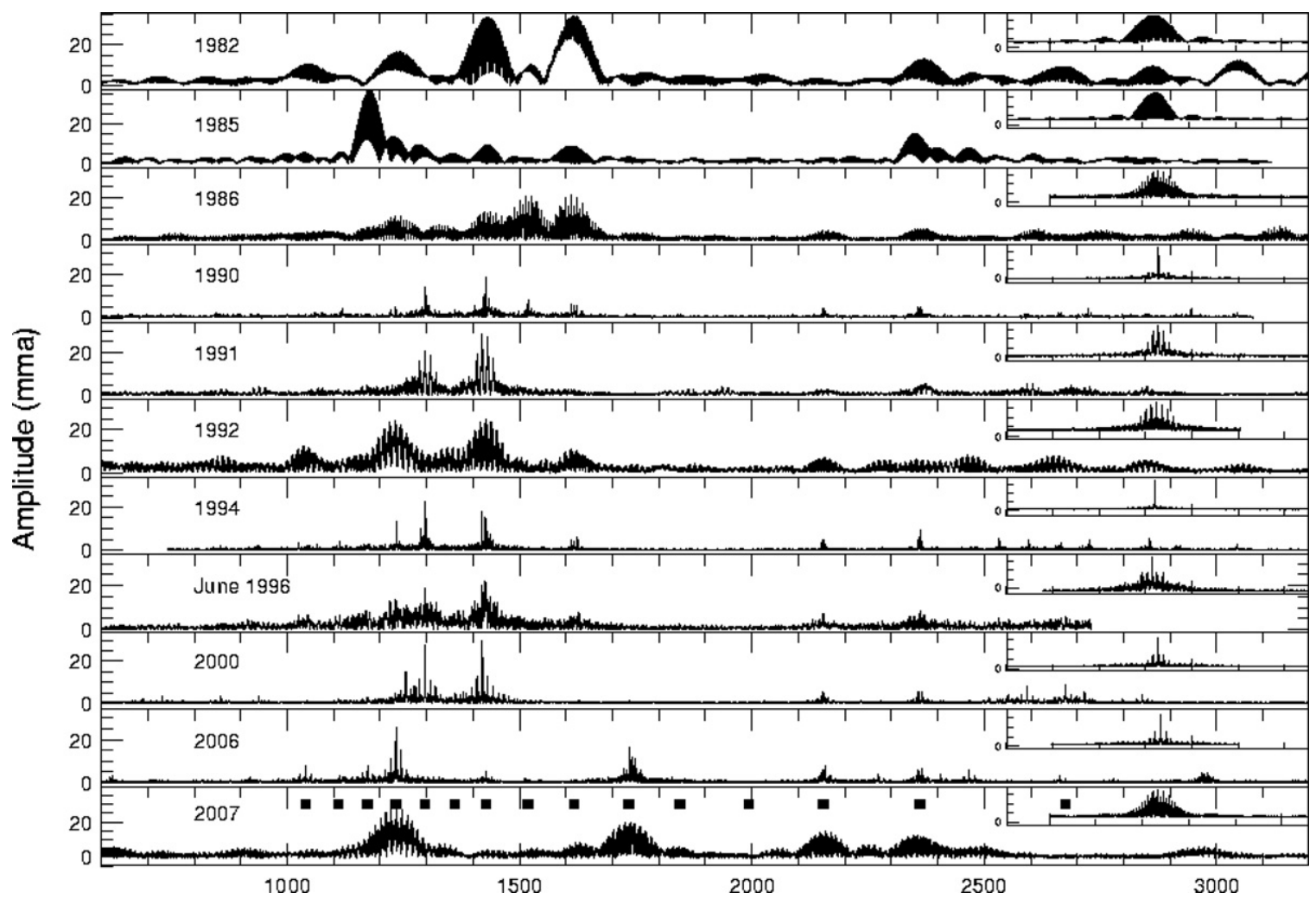

Figure 10. FTs of GD358 from 1982 to 2007, with each spectral window given in the upper right corner of each panel. Each panel spans $2800 \mu \mathrm{Hz}$. The filled squares in the bottom panel mark the predicted locations of GD358's pulsation modes $(l=1)$. GD358 changes its distribution of amplitudes of the excited modes, but the modes are consistently in the same general locations. High- $k$ modes exhibit the largest variation in amplitude. Note the appearance of $k=12$ in 2006.

responding to $l=1$, and quintuplets, corresponding to $l=2$ (see Figures 5 and 6 in Winget et al. 1991).

W94 based the first asteroseismological analysis of GD358 on the identified modes and multiplet structure in the 1990 data set. The frequency splittings within the 1990 multiplets range from 3.5 to $6.5 \mu \mathrm{Hz}$, vary as a function of the radial number $k$, and are not always symmetric with respect to the central frequency. W94 interpreted the trend with $k$ as differential rotation within the star, and used the splitting asymmetry to determine an average magnetic field strength of $1300 \pm 300 \mathrm{G}$. If we assume that GD358's structure is constant in time, then we would expect to find similar multiplet structure in other observing seasons, including XCOV25.

Figure 10 displays FTs for seasons spanning GD358's 1982 discovery to 2007. The 1990 (W94), 1991, 1994, 2000 (K03), and 2006 FTs are from WET runs, while the other FTs are single site, obtained from McDonald Observatory or Mt. Cuba Observatory (2007 season). The large amplitude peaks are consistently found between 1000 and $1800 \mu \mathrm{Hz}$, and individual modes are close to predicted values for $l=1$ (the filled boxes in the last panel of Figure 10 marks the periods of the best fit model from Metcalfe et al. 2003). Using the 2006 data set, we calculated an average period spacing of $38.77 \mathrm{~s}$ using the measured periods of the lowest and highest $k$ modes. This is consistent with results from previous observations (Table 4), demonstrating that GD358's general internal structure is largely unchanged.

We extracted the $k=8,9,15$, and 17 multiplets from the 1990, 1991, 1992, 1994, 1996, 2000, and 2006 seasons, using the techniques discussed in Section 3. The multiplets are plotted in Figures 11, 12, 13, and 14. The low-order $k=8$ and 9 modes consistently display the triplet structure expected for $l=1 g$-modes in the limit of slow rotation (Figures 11 and 12). While the component amplitudes vary from season to season, the frequencies and splitting within the triplets remain roughly the same. The story changes for the $k=15$ and 17 multiplets (Figures 13 and 14). The multiplet structure of these modes exhibit dramatic changes from year to year. If rotational splitting is the dominant mechanism producing the high- $k$ multiplets, we expect them to share the same splitting with other detected modes within the same data set. We have shown that this is not the case (Figure 7).

We now turn our attention to the frequency stability of the $k=8,9,15$, and 17 mode components. Both W94 and K03 report small but significant frequency changes within the identified multiplets, and Figures 11-14 support this finding. For $k=8,9$, and 17 we select the component identified as $m=0$ in W94. For $k=15$, however, we choose $m=-1$ since a peak was present near this frequency more often than the $m=0$ frequency. Figure 15 plots the frequencies of the identified peaks versus time. In all cases, the frequencies shift by several times the statistical errors, where the average error for all the data sets is $\sigma=0.06 \mu \mathrm{Hz}$. The high- $k$ frequencies wander by over $30 \sigma$, much more than the detected $8 \sigma$ changes for $k=8$ and 9 . While these variations are small, they point to very definite changes in the $g$-mode propagation cavity in this star.

Summarizing our results, we find a clear difference between high- and low- $k$ modes. The high $-k$ multiplets are highly variable in amplitude and frequency, and exhibit large changes in multiplet structure. The multiplets within these modes appear to be almost randomly present with the $m=0$ component not necessarily preferred. The low- $k$ modes typically show a stable triplet structure, but the amplitudes of the components are moderately variable. All the multiplets exhibit significant 


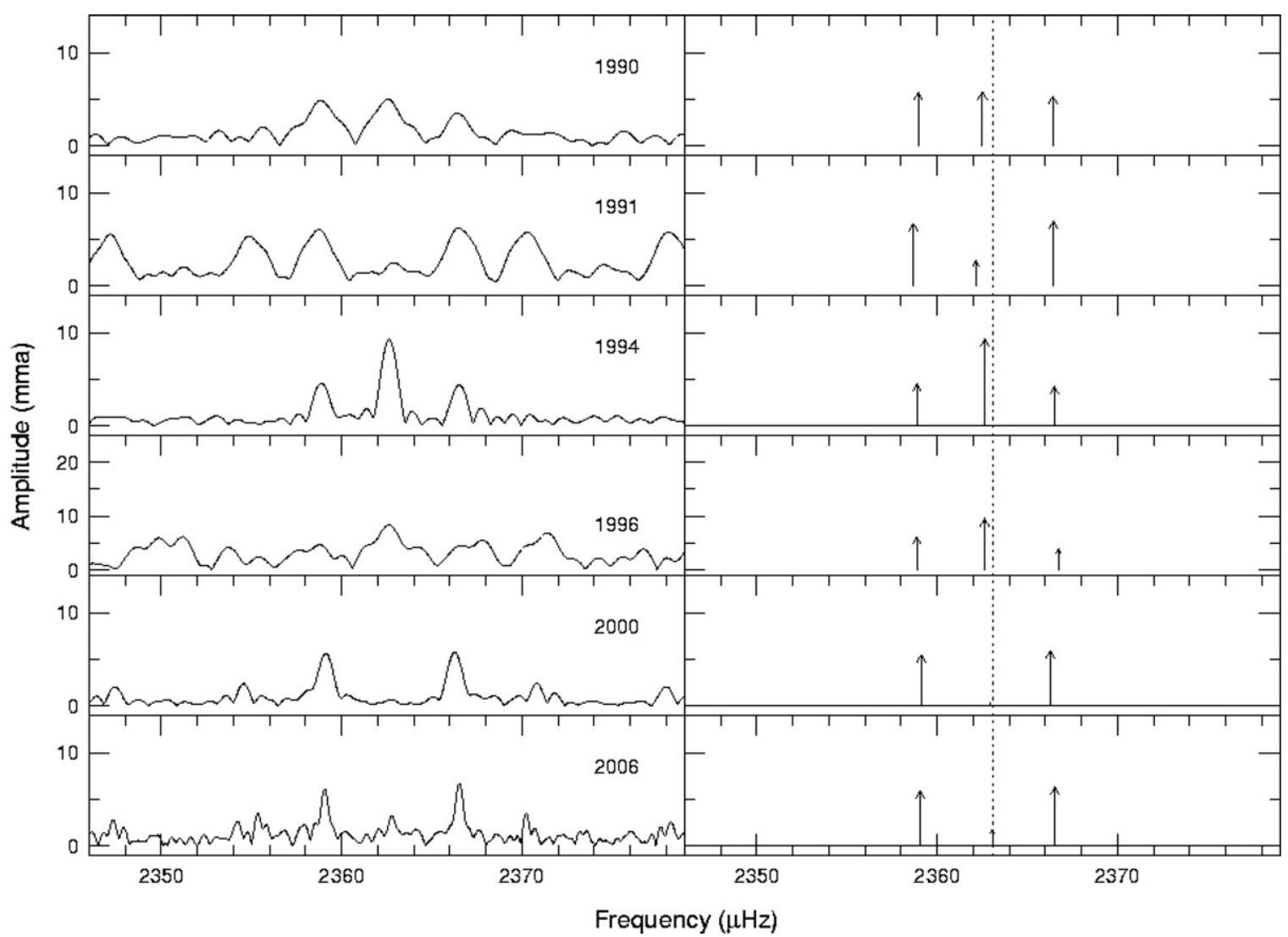

Figure 11. Multiplet structure associated with the $k=8$ mode in GD358 from different observing seasons. The left panels plot the original FTs, and the right panels give the prewhitening results. The largest $1 \sigma$ frequency error bar is $0.06 \mu \mathrm{Hz}(1996)$. Each panel spans $18 \mu \mathrm{Hz}$. The horizontal line through each right panel gives the noise level for that season, and the dotted line marks the 2006 detected frequency.

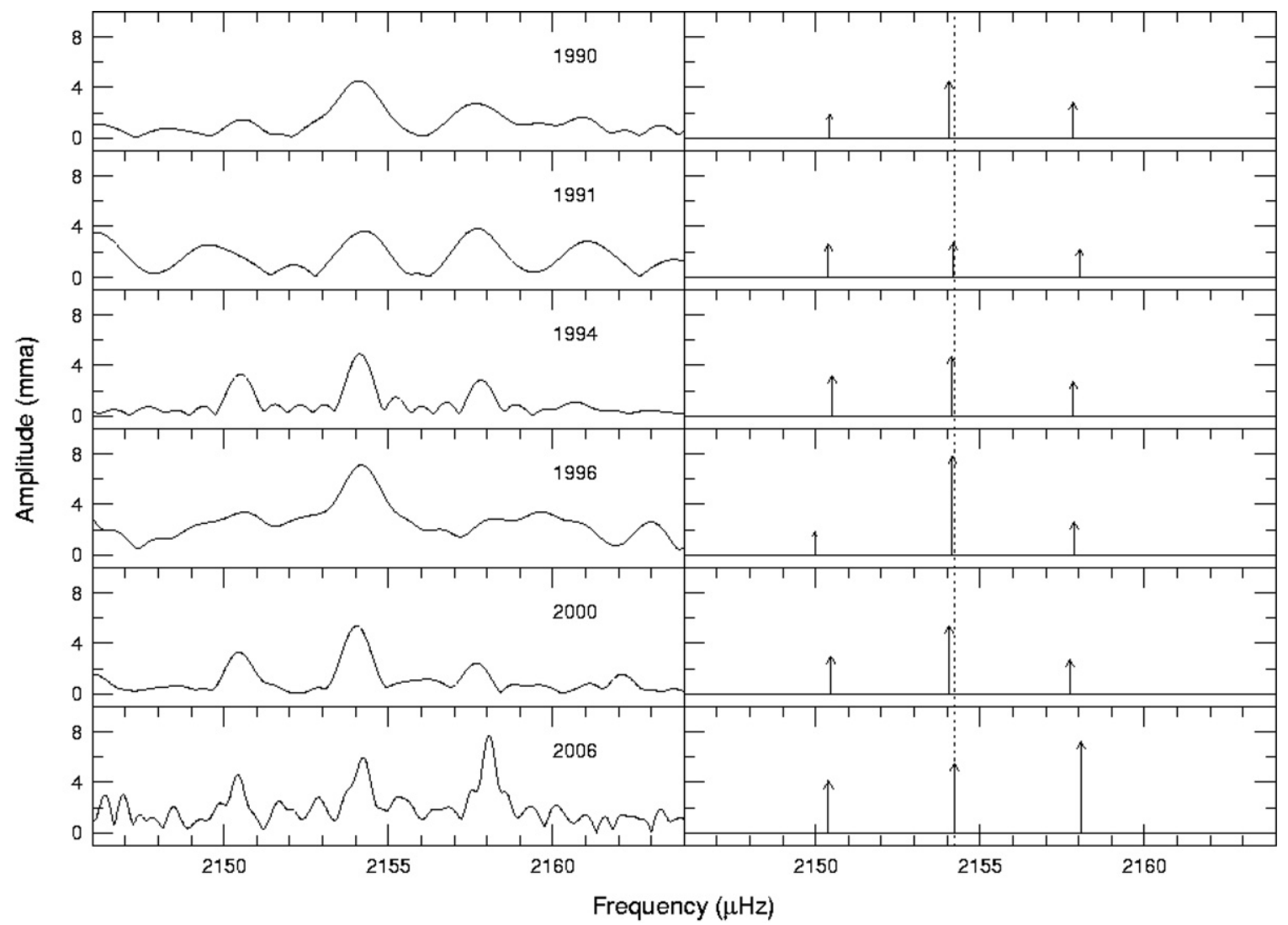

Figure 12. Multiplet structure associated with the $k=9$ mode in GD358 from different observing seasons. The left panels plot the original FTs, and the right panels give the prewhitening results. The largest $1 \sigma$ frequency error bar is $0.06 \mu \mathrm{Hz}(1996)$. Each panel spans $18 \mu \mathrm{Hz}$. The horizontal line through each right panel gives the noise level for that season, and the dotted line marks the 2006 detected frequency. 


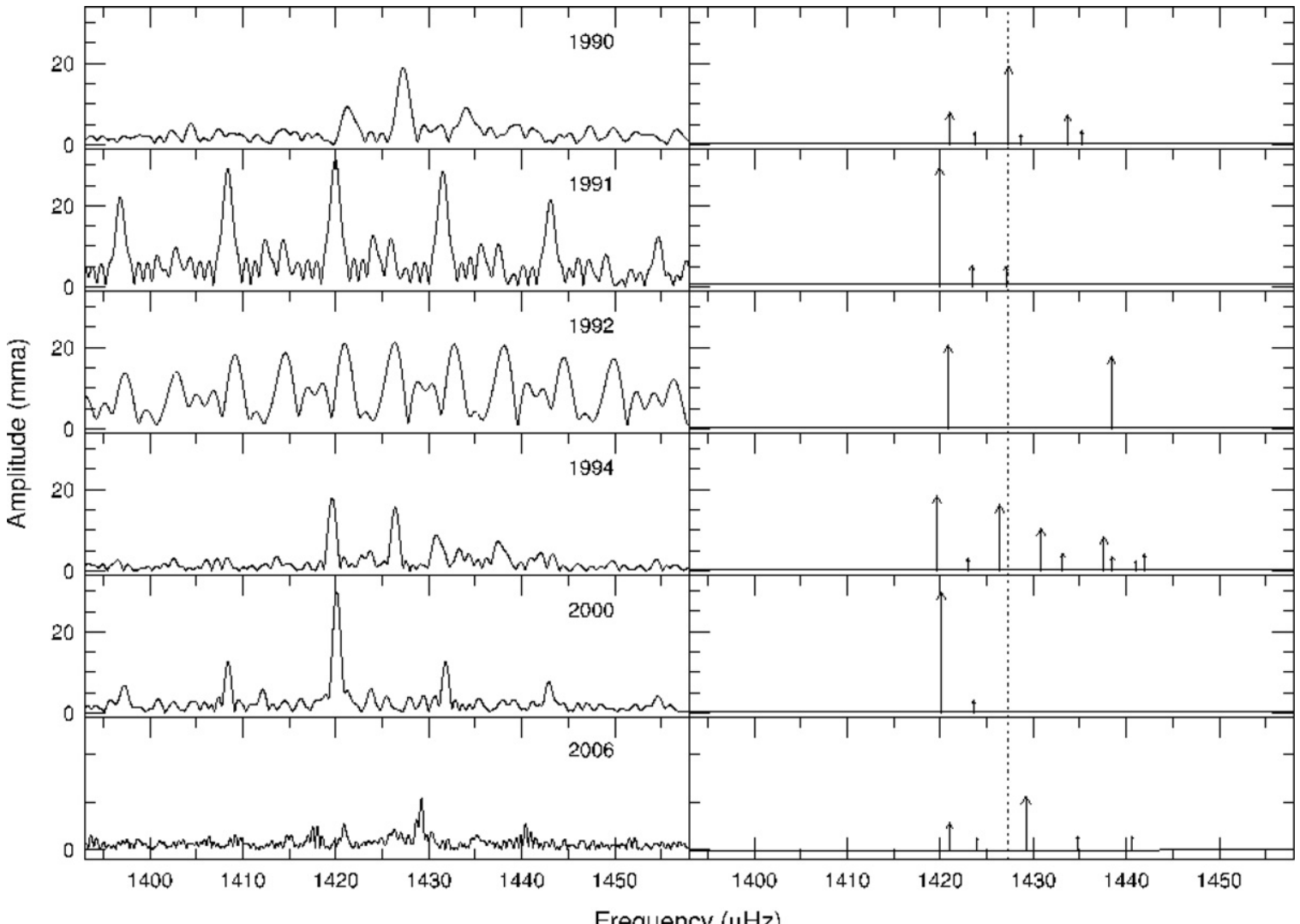

Figure 13. Multiplet structure associated with the $k=15$ mode in GD358 from different observing seasons. The left panels plot the original FTs, and the right panels give the prewhitening results. The largest $1 \sigma$ frequency error bar is $0.06 \mu \mathrm{Hz}$ (1992). Each panel spans $65 \mu \mathrm{Hz}$. The horizontal line through each right panel gives the noise level for that season, and the dotted line marks the 1990 detected frequency.

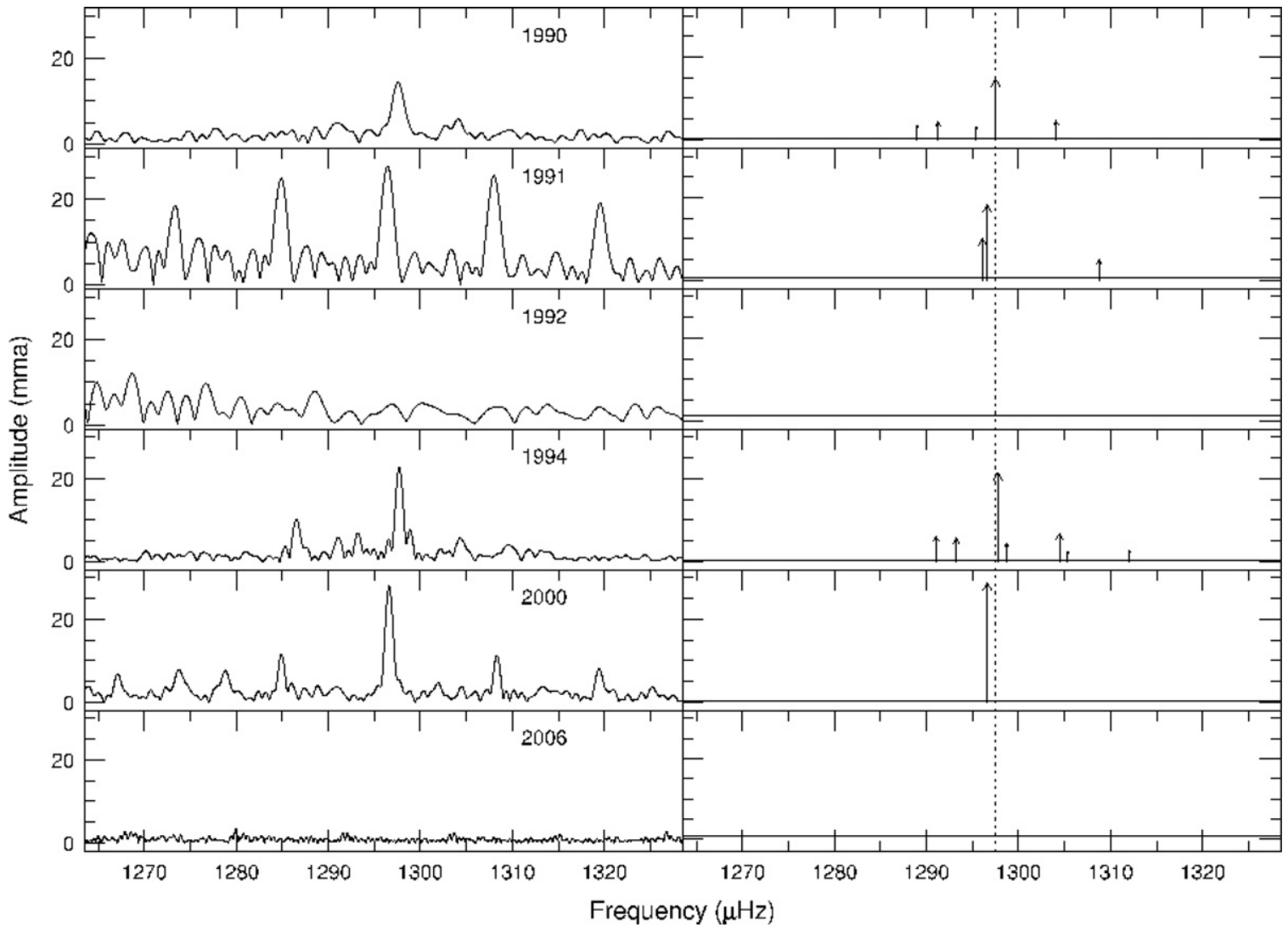

Figure 14. Multiplet structure associated with the $k=17$ mode in GD358 spanning 1990 to 2006. The left panels present the yearly FTs, and the right panels represent the prewhitening results. The largest $1 \sigma$ frequency error bar is $0.06 \mu \mathrm{Hz}(1992)$. Each panel spans $65 \mu \mathrm{Hz}$. The horizontal line through each right panel gives the noise level for that season, and the dotted line marks the 1990 detected frequency. The 2006 peak is difficult to detect due to the $y$ scale. 


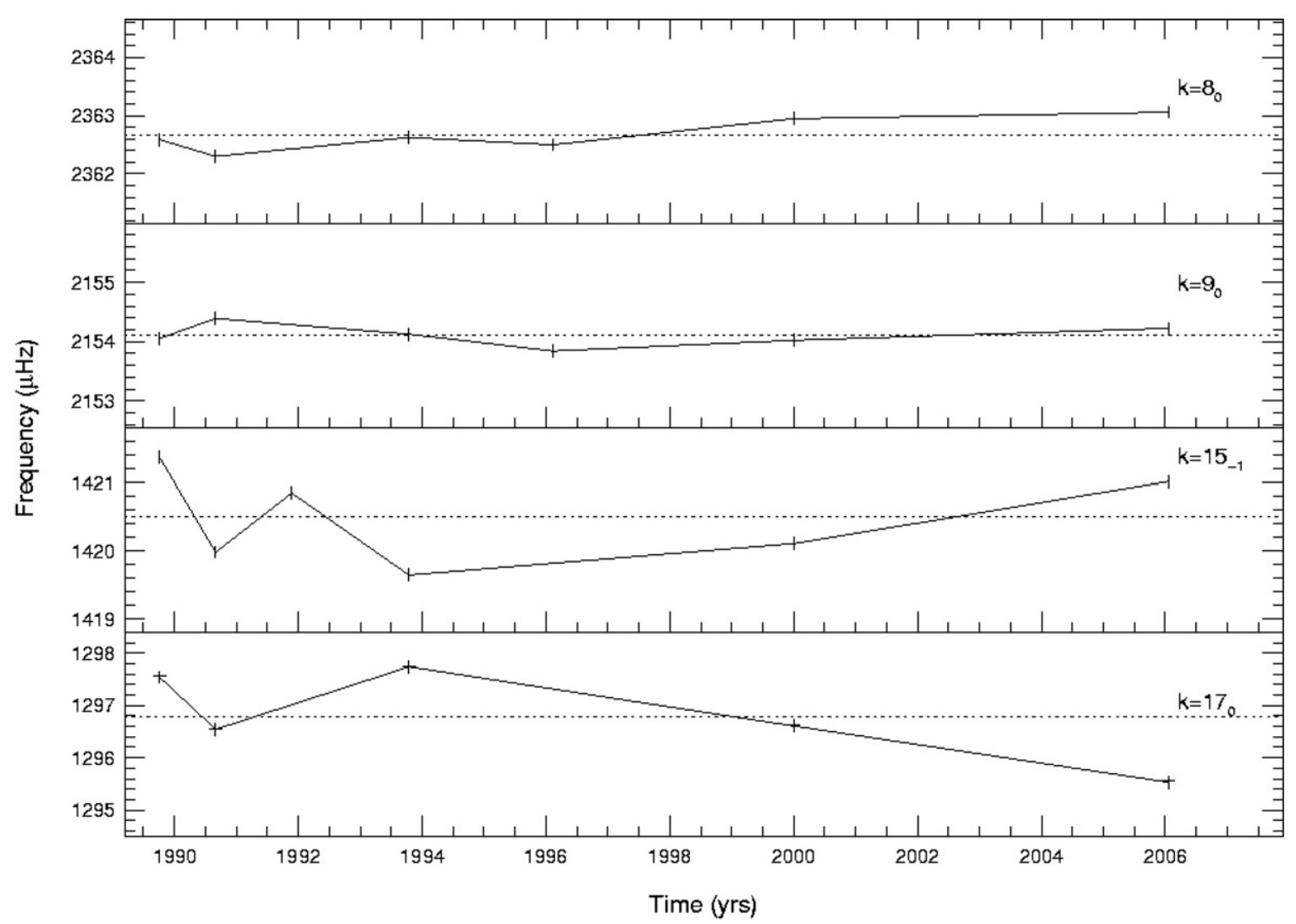

Figure 15. Frequency of the largest component for the $k=17,15,9$, and 8 modes. For $k=17,9$, and 8 , the $m=0$ component was selected. For $k=15$, the $m=-1$ mode was detected more often at larger amplitude. While all modes exhibit some variation in frequency, the high- $k$ modes wander by over $30 \sigma$. The dotted lines mark the reported 1990 frequencies (W94).

wanderings in frequency, with the frequency changes observed in the high- $k$ modes being much larger than those seen in the low- $k$ modes.

\section{IMPLICATIONS FOR GD358}

GD358's 2006 FT contains 27 independent frequencies distributed in 10 excited modes. The locations of the modes agree with previous observations and predicted values for $l=1$ $g$-mode pulsations. The average period spacing $\Delta P_{\mathrm{av}}$, calculated using the lowest $k$ and highest $k$ modes, is identical from 1990 to 2006 (Table 4). As $\Delta P_{\text {av }}$ is fixed by stellar mass and temperature, GD358's basic physical properties have remained unchanged over a timescale of years, as expected. The changes we observe in GD358's pulsation spectra and multiplet structure are not due to gross changes in structure or temperature.

We have shown that the standard model of a pulsating white dwarf in the limit of slow rotation cannot adequately explain the observations of GD358's multiplet structure and behavior. The fine structure of the high- $k$ modes is inconsistent with the expectations of slow rotational splitting, the amplitudes of multiplet components for all $k \mathrm{~s}$ cannot be explained using simple viewing arguments, and the observed frequency variations imply changes in the $g$-mode propagation medium that are much more rapid than expected from evolutionary changes.

The qualitative behavior of the low- $k$ and high- $k$ modes can be explained in the context of a general model. $g$-modes are standing waves of buoyancy in a spherical cavity, and they are thought of as superpositions of traveling waves bouncing back and forth between an inner and an outer turning point. These turning points depend on the mode's period: short period (low- $k$ ) modes have deeper outer turning points than long period (high- $k$ ) modes, so high- $k$ modes sample the outer regions of the star more than low- $k$ modes. If we introduce an additional, non-spherical structure in the star's outer layers, due to magnetic fields or convection, for example, the effect should be much larger on the high- $k$ modes. In effect, GD358 is telling us where this perturbation to its structure must be, but it is still incumbent upon us to diagnose the actual cause.

We do find a clear difference in the photometric behavior of GD358's high- and low- $k$ modes, leading us to consider a connection between the outer layers and its pulsation changes. The following discussion requires that we draw into the mix a remarkable sequence of events that occurred over $\approx 30$ days in 1996. We follow Castanheira et al. (2005) in calling the event the sforzando. In classical music, sforzando is an abrupt change in the character of music, usually accompanied by an increase in volume. We begin with a description of the sforzando. We will examine the implications and connections between our asteroseismic results, the sforzando and two possibilities that may affect GD358's photometric behavior: a surface magnetic field and the convection zone.

\subsection{GD358 in 1996}

In 1996 June, GD358's light curve (third panel, Figure 16) was typically nonlinear, with a dominant frequency of 1297 $\mu \mathrm{Hz}$ corresponding to $k=17$, and a peak to peak intensity variation of $\approx 15 \%$. The $k=9$ and 8 modes were present with amplitudes of 8 and $9 \mathrm{mma}$, respectively. On August 10 (suh-0055, second panel Figure 16), the light curve was again typical, with a $20 \%$ peak to peak intensity varia- 


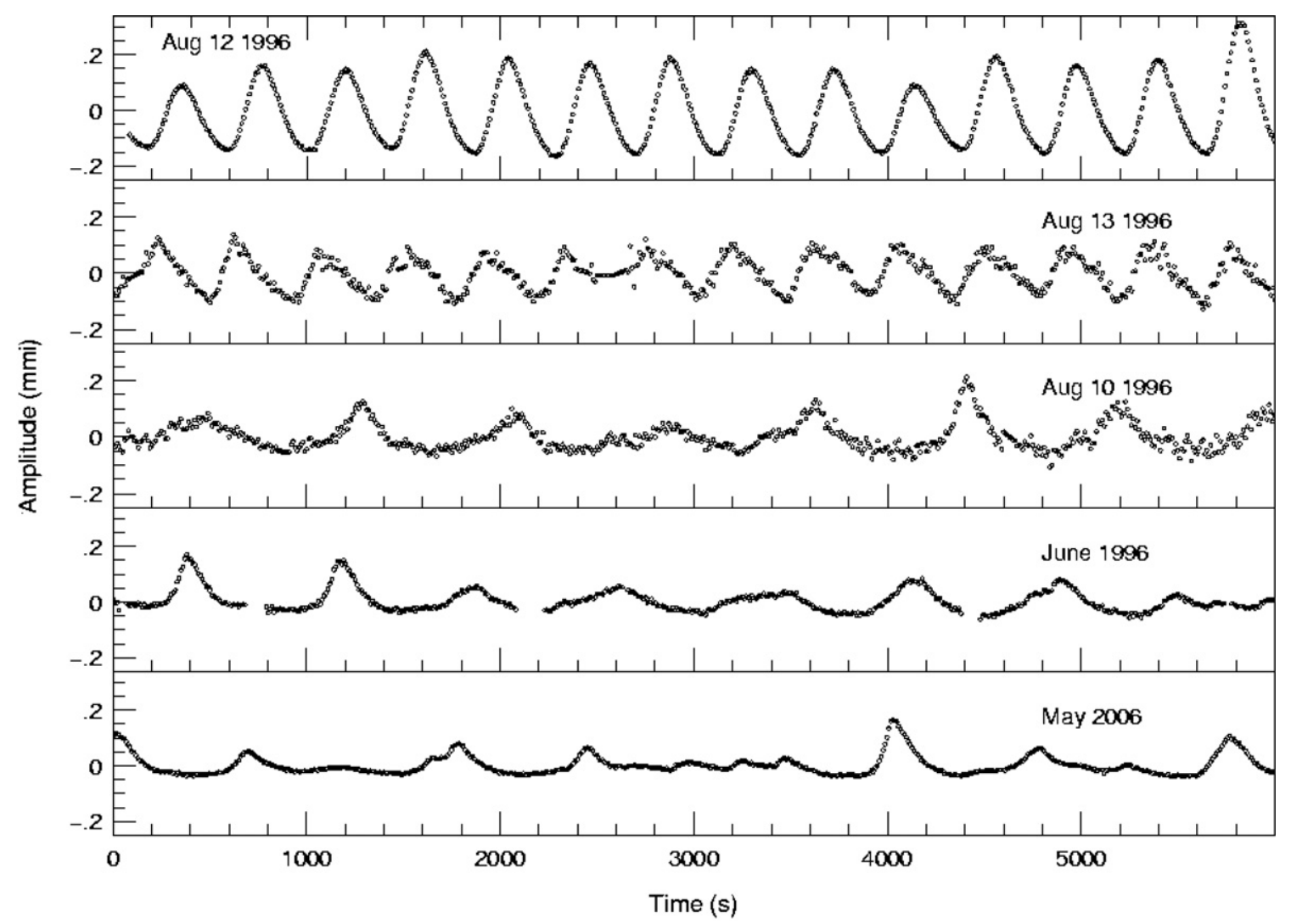

Figure 16. Light curve of GD358 on August 12 (an-0034, top panel) and August 13 (suh-056, second panel), during the 1996 sforzando. On 1996 August 10 (suh-0055, third panel), the light curve was typically nonlinear, as it was in 1996 June (fourth panel), and 2006 May (bottom panel).

tion, and main frequencies consistent with previously observed high- $k$ modes. The $k=9$ and 8 modes had upper amplitude limits of 8 mma. On August 12, 27 hr later, GD358 exhibited completely altered pulsation characteristics (an-0034, top panel Figure 16). The lightcurve was remarkably sinusoidal, with an increased peak to peak intensity variation of $\approx 50 \%$. The $k=8$ mode dominated the lightcurve with a amplitude of $180 \mathrm{mma}$, the highest ever observed for GD358. The $k=9$ mode was also present, with an amplitude of $\approx 20 \mathrm{mma}$. The high- $k$ modes, dominant $27 \mathrm{hr}$ prior, had upper detection limits of $7 \mathrm{mma}$. Over the next $24 \mathrm{hr}$, the $k=8$ mode began to decrease in amplitude (Figure 17). Between Aug 13 and 14, $k=9$ grew in amplitude as $k=8$ decreased, reaching a maximum of $\approx 57 \mathrm{mma}$, and then began to decrease as the $k=8$ mode continued to dissipate. By August 16, a mere $96 \mathrm{hr}$ after the sforzando began, GD358 was an atypically low amplitude pulsator, with power detected in the $k=9$ and 8 modes at amplitudes of $\approx 7 \mathrm{mma}$, and upper limits of less than 5 mma for the high- $k$ modes. By September 10, less than a month after the start of the sforzando, the high- $k$ modes had reappeared, with the dominant mode at $\approx 1085 \mu \mathrm{Hz}$ at an amplitude of 29 mma.

The only observations we have during the maximum sforzando are the PMT photometry (Table 5) observations. Differential photometry does not measure standard stars. However, we can calculate the ratio between the average counts from GD358 and its comparison star, assuming the same comparison star was used each night, and accounting for factors such as extinction and sky variation. In the following, we calculate ratios based on sky subtracted counts obtained within $\pm 1.5 \mathrm{hr}$ of the zenith. We focus on the McDonald $2.1 \mathrm{~m}$ runs an-0034,
Table 5

GD358 1996 August

\begin{tabular}{lcccccc}
\hline \hline Run & $\begin{array}{c}\text { CH1 } \\
\text { GD358 }\end{array}$ & $\begin{array}{c}\text { CH2 } \\
\text { Comp }\end{array}$ & CH1/CH2 & $\begin{array}{c}\text { Time } \\
\text { (BJED) }\end{array}$ & Date & $\begin{array}{c}\text { Length } \\
\text { (hr) }\end{array}$ \\
\hline $\begin{array}{l}\text { McDonald } \\
\text { an-0034 }\end{array}$ & 343727 & 279268 & 1.23 & 2450307.6170160 & Aug 12 & 4.7 \\
an-0036 & 308308 & 290898 & 1.06 & 2450308.6309639 & Aug 13 & 3.2 \\
an-0038 & 269937 & 251923 & 1.07 & 2450309.6362315 & Aug 14 & 3.5 \\
an-0040 & 250813 & 248479 & 1.01 & 2450310.6294625 & Aug 15 & 3.5 \\
$\begin{array}{l}\text { Mt. Suhora } \\
\text { suh-0055 }\end{array}$ & 12786 & 114746 & 0.11 & 2450306.4793479 & Aug 10 & 4.8 \\
suh-0056 & 29408 & 181597 & 0.16 & 2450308.3533174 & Aug 12 & 3.0 \\
suh-0057 & 20271 & 158035 & 0.12 & 2450309.3015281 & Aug 13 & 5.9 \\
suh-0058 & 15974 & 130814 & 0.12 & 2450310.4729899 & Aug 14 & 1.0 \\
suh-0059 & 15434 & 127672 & 0.12 & 2450314.3776294 & Aug 18 & 3.0
\end{tabular}

Notes. Observations made with McDonald $2.1 \mathrm{~m}$. The same comparison star was used in each instance.

an-0036, an-0038, and an-0040. The ratio between GD358 and the comparison star decreased from 1.23 during the height of the sforzando (an-0034, Aug 12) to 1.01 on Aug 15 (an-0040). We also examined observations from Mt. Suhora (Table 5). Suh-0055 was obtained $\approx 24 \mathrm{hr}$ before the sforzando, and the ratio between GD358 and the comparison star was 0.11 . Suh-0056 took place $18 \mathrm{hr}$ after the sforzando maximum but before the pulsations had completely decayed. For suh-0056, the ratio between GD358 and the comparison star increased to 0.16 . For the remaining Mt. Suhora observations, obtained during the 


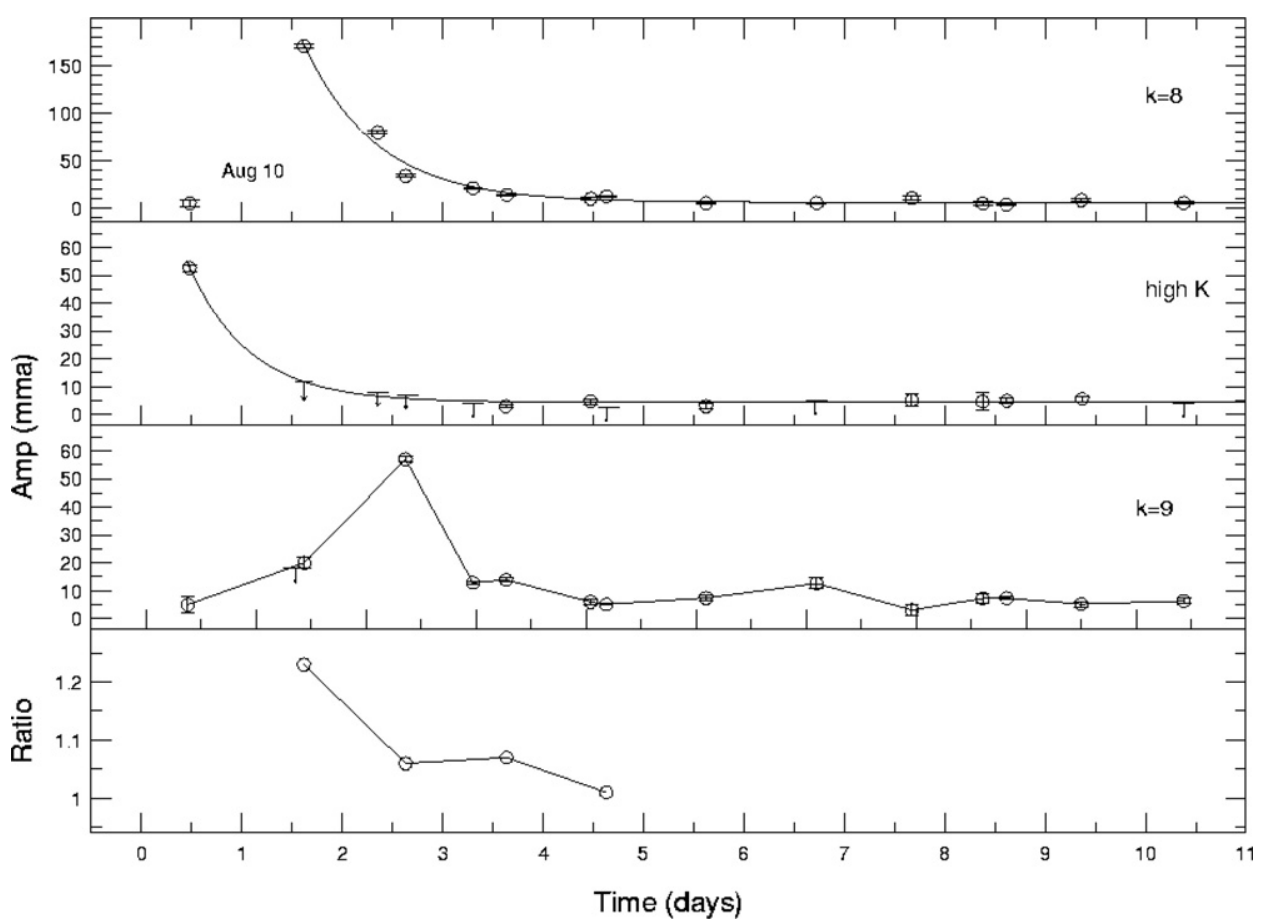

Figure 17. The first three panels give the measured amplitudes for $k=8$ (top panel), the high- $k$ modes, and $k=9$ during the 1996 sforzando. The solid lines give fits using a simple exponential decay model. For $k=8$ and the high- $k$ modes, the damping time was 1.4 days. Note that the "high- $k$ " points do not correspond to any particular $k$ value, but give the highest amplitude (or upper limit) between 1000 and $1500 \mu \mathrm{Hz}$ at that time. The $k=9$ mode grew in amplitude as $k=8$ decayed. The last panel gives the ratio between GD358 and its comparison star from the McDonald $2.1 \mathrm{~m}$ photometry. Note the change in $y$-axis units.

following days, the ratio between GD358 and the comparison star decreased to 0.12 . Observation logbooks at Mt. Suhora demonstrate that the same comparison star, but not the same one used at McDonald, was used each night (Zola 2008). While we cannot pin down any exact numbers due to the nature of differential photometry, the observations of GD358 are consistent with a flux increase in the observed bandpass during the maximum sforzando.

\subsection{Convection and GD358}

The onset of convection goes hand in hand with the initiation of pulsation in both hydrogen and helium white dwarfs (Brickhill 1991; Wu 2000; Montgomery 2005). A primary goal of XCOV25 is to use GD358's nonlinear lightcurve to characterize its convection zone, improving the empirical underpinnings of convective heat transport theory. Our asteroseismological investigation points to evidence that something in the star's outer surface layers is influencing its pulsation modes. The convection zone lies in the outermost regions of GD358, so we are led to consider the relationship between the star's convection zone and its photometric behavior, both typical and during the sforzando.

In general, theoretical studies of convective energy transport are based on the mixing length theory (MLT) (Bohm-Vitense 1958). Originally intended to depict turbulent flows in engineering situations, MLT enjoys success in describing stellar convection (Li \& Yang 2007). It remains, however, an incomplete model with unresolved problems. The actual mixing length is not provided by the theory itself, but is defined as $\left(\alpha H_{p}\right)$, where $H_{p}$ is the pressure scale height, and $\alpha$ is an adjustable variable. This adjustable parameter undermines the ability of theoretical models to make useful predictions. Advances in convection theory include stellar turbulent convection (Canuto \& Mazzitelli 1991; Canuto et al. 1996), which establishes a full range of turbulent eddy sizes. Work is currently underway em- ploying helioseismology to compare MLT and turbulent convection models (Li \& Yang 2007). Our work with convective light curve fitting will also provide important tests for convection theory.

Physical conditions in white dwarf atmospheres differ by orders of magnitude from those in envelopes of main sequence stars like the Sun. For GD358, models indicate that the convection zone is narrow, with a turnover time of $\approx 1$ second and a thermal relaxation timescale of $\approx 300 \mathrm{~s}$ (Montgomery 2008). The pressure scale height is small, limiting the vertical height of the convective elements. Montgomery \& Kupka (2004), employing an extension of stellar turbulent convection, calculate that $40-60 \%$ of GD358's flux is carried by convection, depending on the adopted effective temperature.

Convective lightcurve fitting is based on the assumption that the convection zone, by varying its depth in response to the pulsations, is responsible for the nonlinearities typically observed in GD358's lightcurves (Montgomery 2005). During the sforzando, within an interval of $27 \mathrm{hr}$, GD358's lightcurve became sinusoidal, arguing that the convection zone was inhibited, at least as sampled by the $k=8$ mode. For example, if this large amplitude mode was the $m=0$ member of the triplet, then its brightness variations would be predominantly at the poles and not the equator. Thus, all that is required to produce a sinusoidal lightcurve is a mechanism to inhibit convection near the poles.

How could convection in any star be decreased on such short timescales? The obvious method, but not necessarily the most physical, is to raise GD358's effective temperature, forcing it to the blue edge of the instability strip, and decreasing convection. Such a temperature increase has the observable consequence of increasing the stellar flux. A flux increase of $20 \%$ in our effective photometric bandpass would produce an equivalent change in the bolometric flux of $\approx 40 \%$, corresponding to a temperature change of $\approx 2200 \mathrm{~K}$. The sforzando photometry is consistent 
with such an increase in flux, and Weidner \& Koester (2003) find that $\mathrm{T}_{\mathrm{eff}} \approx 27,000 \mathrm{~K}$ is required to simulate GD358's light curve during the maximum sforzando. Castanheira et al. (2005) use FOS spectroscopy of GD358 obtained on 1996 August 16 to determine $\mathrm{T}_{\text {eff }}=23900 \pm 1100 \mathrm{~K}$, a number not inconsistent with previous observations (Beauchamp et al. 1999). However, the FOS spectra were acquired after the pulsations had greatly decreased in amplitude (Figures 16 and 17). It is possible that GD358 had cooled by this point. Recent theoretical evidence also indicates that simply "turning off" convection would have minimal observable effects on GD358's spectrum (Koester 2008).

The connection between convection and pulsation during the sforzando may also depend on the origin of the event. Was the sforzando a short-term change, such as a collision, or a more global event intrinsic to GD358? The growth and dissipation of modes during the sforzando could be connected to pulsation growth timescales, or may be governed by a completely different mechanism. Pulsation theory predicts that growth rates for high$k$ modes are much larger than for low- $k$ modes. This simply means that high- $k$ modes are easier to excite and have growth times (and decay times) of days rather than years (Goldreich \& Wu 1999). This makes sense for GD358, where we typically find the high- $k$ modes to be much more unstable in amplitude over time than $k=9$ and 8 (Figure 10). Figure 17 plots the amplitudes for the $k=8, k=9$, and "high- $k$ " modes during the sforzando. We note that the "high- $k$ " points do not correspond to any particular $k$ value, but give the highest amplitude (or upper limit) between 1000 and $1500 \mu \mathrm{Hz}$. We fit the observed amplitudes for $k=8$ and the high $k$ s with a simple exponential decay model $A(t)=A_{0} e^{-t / \tau_{d}}+c$, where $\mathrm{A}(\mathrm{t})$ is the amplitude at time $t$ and $\tau_{d}$ is the damping constant. Our fits give a damping timescale of $\tau_{d}=1.5 \pm 0.2$ days for both the $k=8$ mode and the high $k$ s. The $k=9$ mode, which actually grew in amplitude later, as $k=8$ decayed, has an upper limit of $\tau_{9^{d}}<1.1$ days. We only have a few points measuring the growth timescale $\left(\tau_{g}\right)$ of $k=8$ and 9, so we place an upper limits for $k=8$ of $\tau_{8^{g}} \leqslant 1.2$ days. For $k=9$, we find an upper limit of $\tau_{9 g} \leqslant 2.2$ days. These timescales are all roughly equivalent, are all much longer than the dynamical timescale, and not distributed as predicted by theoretical pulsation growth rates. The results for $k=9$ and 8 are much shorter than the expected pulsation damping timescale of about two months (Montgomery 2008a), arguing that the source of the sforzando was capable of interacting with the pulsations to produce changes on faster timescales than theoretical growth rates.

Our purpose in entering this discussion of the sforzando was to provide insight into connections between convection and pulsation in GD358. We find evidence that convection diminished during the sforzando. The decreased convection was accompanied by the rapid disappearance of GD358's high- $k$ modes and the transfer of energy to the $k=8$ and 9 modes. The photometry is consistent with a temperature increase, which would inhibit convection. The sudden transfer of energy from the high- $k$ modes to $k=9$ and 8 argues that a mechanical (radial or azimuthal) or thermal structural change altered GD358's outer layers, modifying mode selection. We find that the growth/damping timescales during the sforzando for the $k=9$ and 8 modes do not agree with the expectations of pulsation theory, and may be governed by a completely different mechanism.

Magnetic fields have long been known to be capable of inhibiting convection. For example, localized fields associated with sunspots in the Sun are observed to inhibit convection in the surrounding photosphere (Biermann 1941; Houdek et al. 2001). Winget (2008) estimates that the magnetic decay time at the base of the convection zone is $\approx 3$ days. In the next section, we investigate the possible implications of magnetic fields on GD358 and its pulsations.

\subsection{Magnetic Fields}

Our current understanding of magnetic fields in stars like the Sun invokes the dynamo mechanism, which is believed to function in the narrow region where rotation changes from the latitudinal rotation of the outer convective layers to spherical rotation in the radiative zone (Morin et al. 2008). The solar magnetic field consists of small, rapidly evolving magnetic elements displaying large scale organization, with a cycle time of 22 years during which the polarity of the field switches. Localized fields, with strengths hundreds of times stronger than the global field, exert non-negligible forces on charged particles, influencing convective motion. Solar pulsations are observed to vary in frequency with the solar cycle in these regions (Schunker $\&$ Cally 2006). Although the exact mechanism is uncertain, the frequency shifts are interpreted as representing change in the Sun's internal structure or driving (Woodward \& Noyes 1985; Kuhn 1998; Houdek et al. 2001).

A pulsating white dwarf represents an extreme environment. The atmosphere and the convection zone are thin, the surface gravity orders of magnitude higher, and differential rotation may or may not play a role (Kawaler et al. 1999). The magnetic field in a typical white dwarf is confined to the nondegenerate outer layers. The field may dominate near the surface, but deeper in the atmosphere, gas pressure will prevail. Drawing an analogy with the Sun, a surface magnetic field in GD358 probably has an associated cycle time similar to the solar cycle. Hansen et al. (1977) and Jones et al. (1989) discuss this topic from a theoretical perspective, predicting cycle times ranging from two to six years.

What are the observable consequences of a magnetic field on white dwarf pulsations? Jones et al. (1989) find that, in the presence of a weak field, defined as strong enough to perturb but not dominate mass motions, multiplet frequencies are increased with respect to the central mode in a manner proportional to $\mathrm{m}^{2}$. The $m=0$ mode is also shifted, unlike the case for rotation. Each $g$-mode pulsation samples the magnetic field at a different depth. A logical expectation is that, for low- $k$ modes with reflection points below the convection zone, we can treat the magnetic field as a perturbation, while for high- $k$ modes such a treatment is not valid.

Our best candidates to investigate the influence of a magnetic field on multiplet structure are GD358's $k=9$ and 8 modes. These two modes are consistent with rotationally split triplets and we can examine their multiplet structure in detail over 16 years of observations (Figures 18 and 19). From 1990 to $2006, k=9$ had an average multiplet width of $7.7 \pm 0.1 \mu \mathrm{Hz}$, while $k=8$ is similar, at $7.5 \pm 0.1 \mu \mathrm{Hz}$. Figures 18 and 19 show that the complete multiplet width and the splittings with respect to the central mode wander up to $0.5 \mu \mathrm{Hz}$ from the average values. Both Figures 18 and 19 reveal a dramatic change during the sforzando. In June 1996, both multiplets increased in total width by $\approx 1 \mu \mathrm{Hz}$. Prior to August 1996, the retrograde splitting $(0,-1)$ for $k=8$ was consistently smaller by $\approx 0.2 \mu \mathrm{Hz}$ than the prograde splitting $(0,+1)$. After 1996 August, the retrograde splitting became consistently larger by $\approx 0.5 \mu \mathrm{Hz}$ than the prograde splitting and has remained so through 2007 , at the 


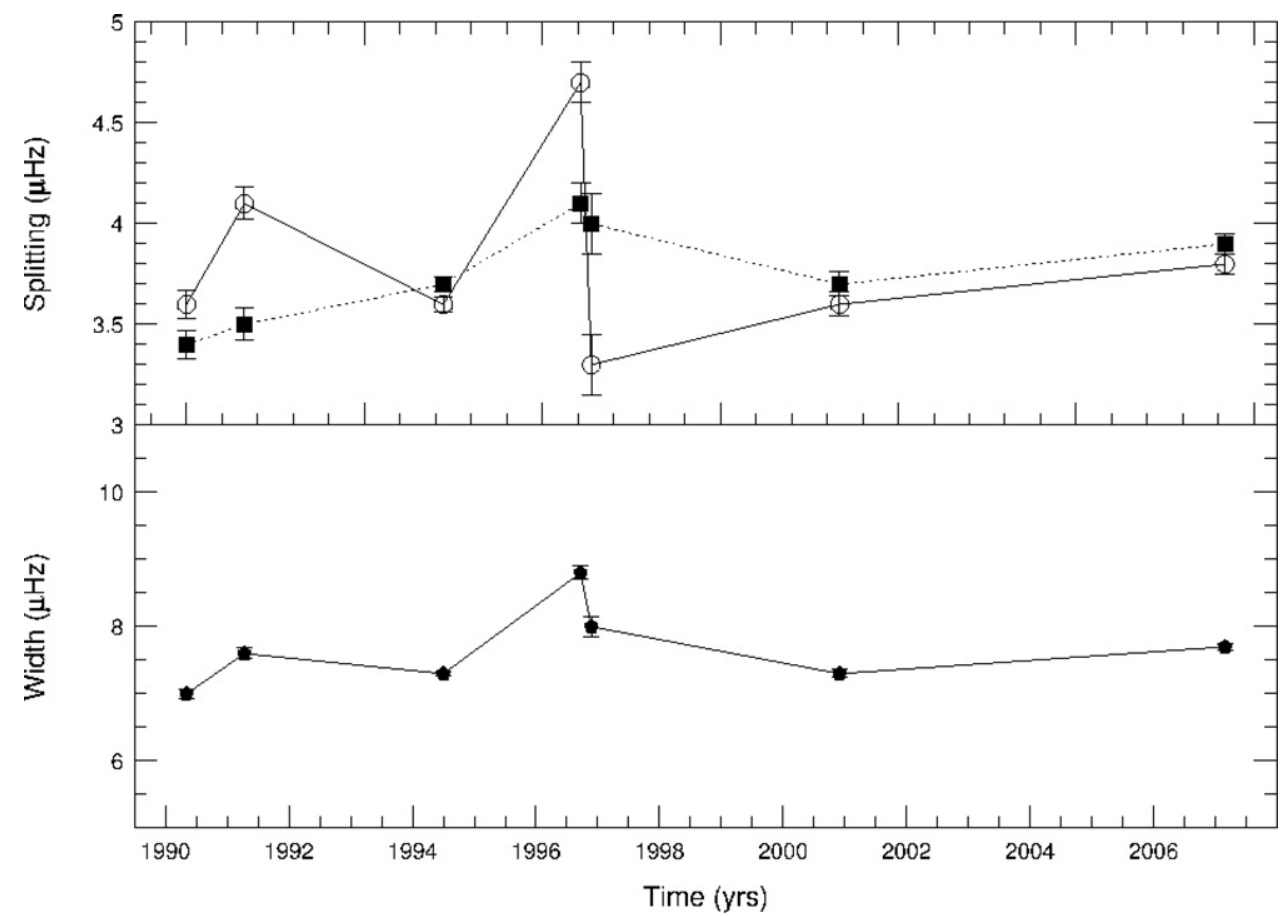

Figure 18. Multiplet structure within the $k=9$ multiplet. The $x$-axis is time in years, and the $y$-axis is in $\mu \mathrm{Hz}$. The bottom panel plots the width of the entire multiplet over time. The top panel examines the $m=0,+1$ (dotted line) and $m=0,-1$ (solid line).

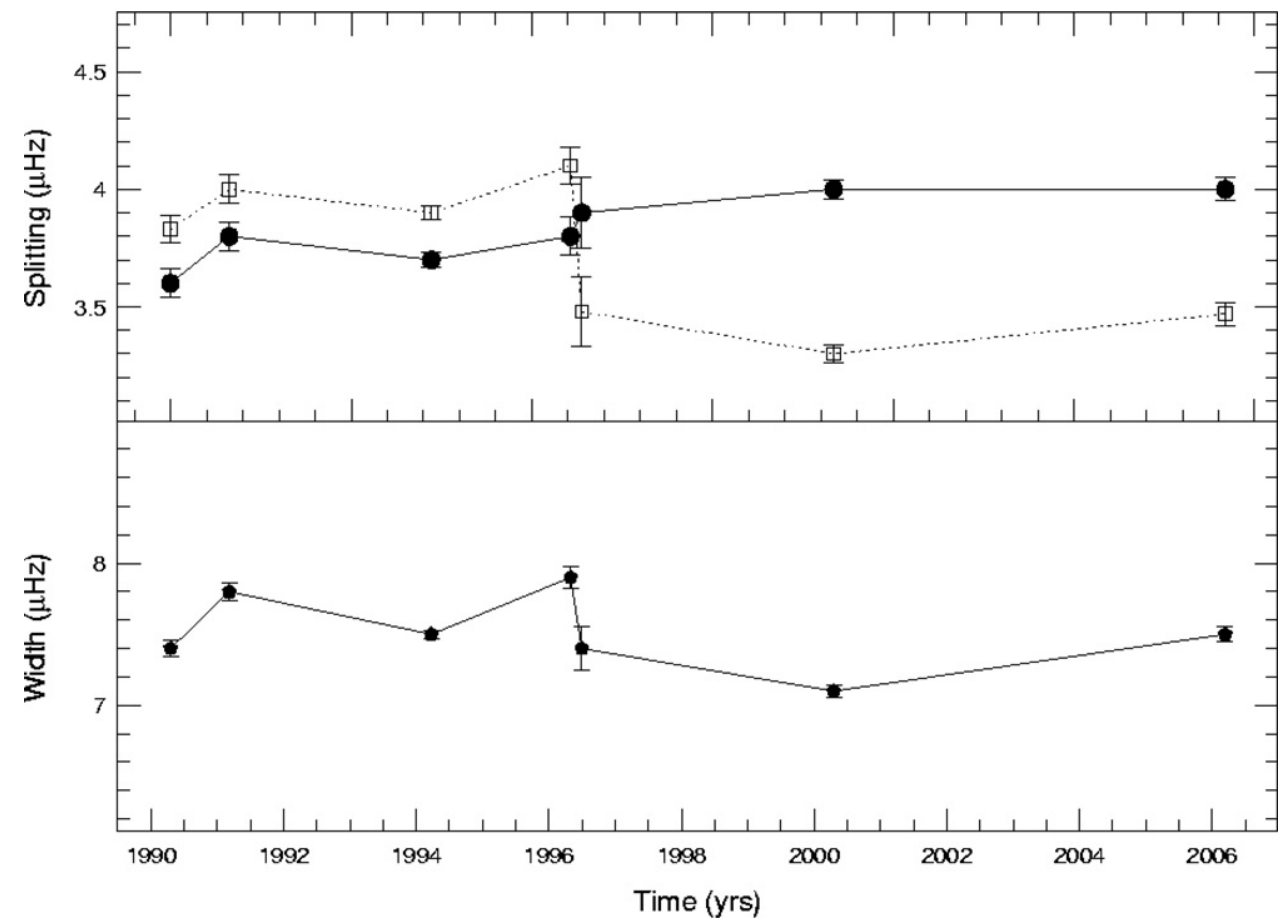

Figure 19. Multiplet structure with $k=8$ multiplet. The $x$-axis is time in years, and the $y$-axis is in $\mu \mathrm{Hz}$. The bottom panel plots the width of the entire multiplet over time. The top panel examines the $m=0,+1$ (dotted line) and $m=0,-1$ (solid line).

resolution of our observations. The case is not so straightforward for $k=9$, but this mode clearly shows a large change in 1996, especially for the $(0,-1)$ splitting (Figure 18$)$. In the previous section, we questioned whether the sforzando was a short-term event or a global, enduring change. This analysis of $k=9$ and 8 points toward a long-term change in GD358's resonance cavity. The changes we observe in the multiplet structure could be explained by a change in magnetic fields.
A magnetic field also introduces an additional symmetry axis. Our analysis of GD358's harmonics and combination frequencies hints that something is disrupting its azimuthal symmetry. It is conceivable that high- $k$ modes would align more or less with the magnetic field, while the low- $k$ modes align with the rotation axis. $\mathrm{Wu}$ (2001) \& Yeates et al. (2005) do not explore the possibility of multiple axes or the effects of a magnetic field on nonlinear mixing by the convection zone. Future work could 


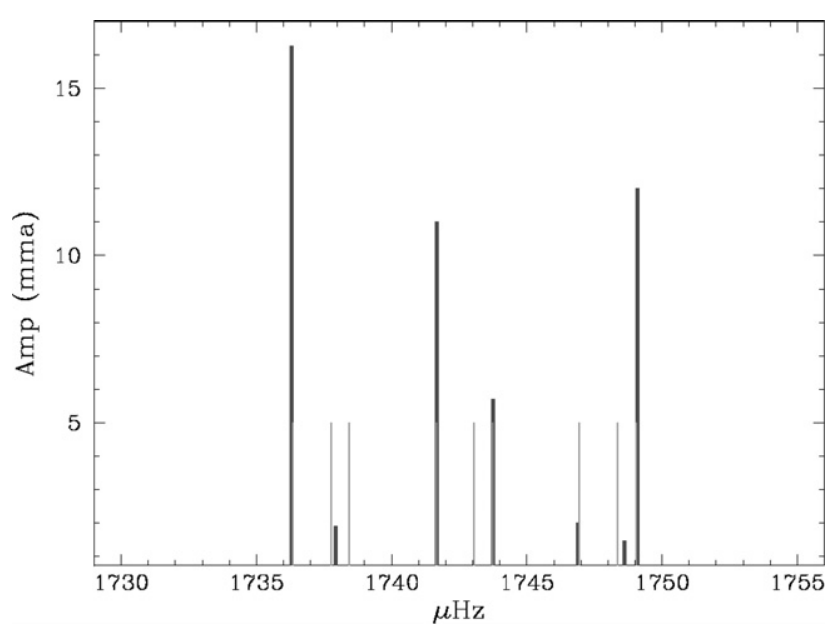

Figure 20. Fit to $k=12$ components assuming the dominant splitting mechanism is a magnetic field using the model of Unno et al. (1979) rather than rotation. The black lines are the observed multiplet components, including several below our detection limits that are not listed in Table 2. The light gray lines are the theoretical predictions. Theoretical predictions overlap the four largest amplitude peaks, although they are difficult to distinguish in the figure.

explain why GD358's modes do not combine as expected by simple theory.

The presence of the $k=12$ mode offers a final hint of a possible surface magnetic field. The second largest mode in the $2006 \mathrm{FT}, k=12$ appears in a region that is normally devoid of significant power. If, for this mode, the magnetic field can no longer be treated as a perturbation, we would expect a multiplet with $(2 l+1)^{2}$ components, as opposed to the three expected from rotation (Unno et al. 1979). For $l=1$, this corresponds to nine magnetic components. We do find six peaks above our $4 \sigma$ limit, with three additional peaks slightly just our criteria. Using the magnetic model of Unno et al. (1979), we are able to match the frequencies of the $k=12$ mode components, but not the amplitudes (Figure 20).

\section{SUMMARY AND CONCLUSIONS}

GD358 is the best studied of the DB pulsators, yet our work shows that this object is by no means completely understood. The 2006 XCOV25 observations were obtained with the goal of using GD358's nonlinear lightcurve to characterize its convection zone, but our initial asteroseimological analysis of the data set reveals a great deal of interesting information about GD358's pulsational behavior. Our investigation began with an analysis of GD358's 2006 XCOV25 FT. We explored the identified modes, combination frequencies, and multiplet structure. Difficulties in identifying the $m$ components of the 2006 mode multiplets, both directly and indirectly using the combination frequencies, lead us to examine the multiplet structure in detail over time. Our investigation expanded to include observations of GD358 over the 24 years since its discovery, focusing on the multiplet structure, frequency stability, and photometric behavior of GD358. We summarize our results concerning GD358's pulsation properties:

1. The 2006 FT contains 27 independent frequencies distributed in 10 modes $(k=21,19,18,17,15,14,12,11,9$, and 8). The dominant frequency is at $1234 \mu \mathrm{Hz}(k=18)$ with an amplitude of 24 mma.

2. We find significant power at $k=12$, a region of the FT previously devoid of significant power.
3. The frequency location of each mode in the 2006 FT is consistent with previous observations and theoretical predictions, assuming $l=1$.

4. The $k=9$ and 8 modes exhibit triplets in agreement with theoretical predictions for $l=1$ in the limit of slow rotation. The amplitudes and frequencies of the components exhibit some variability over time, but much less than the high- $k$ modes.

5. Our analysis of GD358's high- $k$ multiplets over time reveal that they are variable in multiplet structure, amplitude, and frequency. The variability and complexity of the high- $k$ multiplets cannot be interpreted simply as $l=1$ modes in the limit of slow rotation.

6. We cannot provide $m$ identifications for most of the multiplet components in the $2006 \mathrm{FT}$, with the exception of $k=$ 9 and 8.

7. The 2006 FT contains a rich assortment of combination frequencies. They are potential tools for identifying $m$ values and orientation for each mode. However, their amplitudes do not agree with theoretical predictions from Wu (2001).

8. The $k=9$ and 8 multiplet amplitudes and presence/absence of harmonics cannot be explained by simple geometric viewing arguments, and argue that something is interrupting GD358's azimuthal symmetry.

9. The linear, sinusoidal shape of the lightcurve indicates that GD358's convection zone was diminished during the 1996 sforzando.

10. Photometry taken during the maximum sforzando is consistent with a flux increase in the effective bandpass.

11. We find damping/growth timescales during the sforzando that are not consistent with the expectations of pulsation theory.

12. Changes in the splittings of the $k=9$ and 8 multiplets indicate that some mechanism, perhaps a magnetic field, induced a long-term change in the multiplet structure of these modes during the sforzando.

Our investigation raises a number of interesting implications for our understanding of the physics of GD358, many beyond the scope of this paper, and some blatantly skirting the realm of speculation. While we cannot pretend that our investigation has unearthed unshakable evidence thereof, we do find tantalizing indications pointing to connections between convection, magnetic fields, and pulsation in GD358. We suggest future investigations:

1. Theoretical investigation of the relationship/connection between magnetic fields, convection, and pulsation will increase our understanding of GD358's photometric behavior. The typical static model of a pulsating white dwarf is too limiting. The dynamic model must accommodate the observed changes in pulsation frequencies and multiplet structure.

2. GD358's multiplets do not conform to theoretical expectations based on rotation. Other mechanisms must be considered. Examination of the influence of a nonspherical asymmetry in the outer layers on the multiplet structure is required to understand GD358's multiplets. Identification of spherical degree by multiplet structure alone should be highly suspect in any large amplitude pulsator.

3. Further work on the theoretical aspects of combination frequencies should explore the effects of multiple symmetry axes and the effects of a magnetic field on nonlinear mixing by the convection zone. 
4. Detailed investigation of the FOS spectrum obtained during the sforzando for possible metals may provide insight into the mechanism producing the event.

5. Theoretical predictions for growth and damping rates have been calculated using static models. The timing of the growth and decay of $k=8$ and 9 during the sforzando indicates interaction between these two modes. A theoretical examination of growth rates in the presence of other modes, including the interaction of a mode with itself, is necessary to better understand both the growth and dissipation of modes during the sforzando and typical mode selection in all large amplitude DB and DA pulsators.

6. A detailed examination of behavior of white dwarf pulsators, both low and high amplitude, spanning both the DA and DB instability strips (and hence a range of convective depths) will improve our understanding of convection's role in mode selection.

7. Theoretical work is needed to improve the treatment of convection. Lightcurve fitting of GD358 and other pulsators is an important step towards that goal.

8. Continued observation of GD358 will better define its dynamic behavior. We would like to observe another sforzando.

The practice and theoretical development of asteroseismology of GD358 and other pulsating stars continues to reward us with a rich scientific return. Our focus here on GD358 shows us that stellar seismology can challenge our current paradigm of the interior and behavior of pulsating white dwarfs.

The Delaware Asteroseismic Research Association is grateful for the support of the Crystal Trust Foundation and Mt. Cuba Observatory. DARC also acknowledges the support of the University of Delaware, through their participation in the SMARTS consortium. This work is further supported by the Austrian Fonds zur Förderung der wissenschaftlichen Forschung under grant P18339-N08. We would like to thank the various Telescope Allocation Committees for the awards of telescope time. We also acknowledge the assistance of J. Berghuis, M. David, H. Wade, and U. Burns with the Hawaii 0.6m. D.E.M acknowledges his work as a part of research activity of the Astrophysical Research Center for the Structure and Evolution of the Cosmos (ARCSEC) which is supported by the Korean Science and Engineering Foundation.

Facilities: MCAO:0.6 m, McD:2.1 m, KPNO:2.1 m, UH:0.6 m, BOAO:1.8 m, Lulin:1.8 m, Beijing:2.16 m, Maidanek:1.0 m, Peak Terskol:2.0 m, Moletai:1.65 m, Mt. Suhora:0.6 m, Konkoly:1.0 m, Vienna:0.8 m, Tübingen:0.8 m, OHP:1.93 m, NOT, ING:Herschel, LNA:1.6 m, CTIO:0.9 m, SOAR

\section{REFERENCES}

Alves, V. M., et al. 2003, Balt. Astron., 12, 33

Beauchamp, A., Wesemael, F., Bergeron, P., Fontaine, G., Saffer, R., Liebert, J., \& Brassard, P. 1999, ApJ, 516, 887

Biermann, L. 1941, Mitt. Ges., 76, 1941

Bigot, L., \& Dziembowski, W. A. 2002, A\&A, 392, 235

Bohm-Vitense, E. 1958, Z. Astrophys., 46, 108

Bradley, P. A., \& Winget, D. E. 1994, ApJ, 430, 850
Breger, M., et al. 1993, A\&A, 271, 482

Brickhill, A. J. 1991, MNRAS, 251, 273

Brickhill, A. J. 1992, MNRAS, 259, 519

Canuto, V. M., Goldman, I., \& Mazzitelli, I. 1996, ApJ, 473, 550

Canuto, V. M., \& Mazzitelli, I. 1991, ApJ, 370, 295

Castanheira, B., Kepler, S. O., Nitta, A., Winget, D. E., \& Koester, D. 2005, A\&A, 432, 175

Di Mauro, M. P., Christensen-Dalsgaard, J., Kjeldsen, H., Bedding, T. R., \& Paterno, L. 2003, A\&A, 341

Dolez, N., Vauclair, G., \& Kleinman, S. J., et al. 2006, A\&A, 446, 237

Goldreich, P., \& Wu, Y. 1999, ApJ, 511, 904

Handler, G., Romero-Colmenero, E., \& Montgomery, M. H. 2002, MNRAS, 335,399

Hansen, C. J., Cox, J. P., \& Van Horn, H. M. 1977, ApJ, 217, 151

Houdek, G., et al. 2001, MNRAS, 327, 483

Ising, J., \& Koester, D. 2001, A\&A, 374, 116

Jones, P. W, Pesnell, W. D., Hansen, C. J., \& Kawaler, S. D. 1989, ApJ, 336, 403

Kanaan, A., Kepler, S. O., \& Winget, D. E. 2002, A\&A, 389, 896

Kanaan, A., et al. 2005, A\&A, 432, 219

Kawaler, S., Sedekii, T., \& Gough, D. 1999, ApJ, 516, 349

Kepler, S. O., et al. 2003, A\&A, 401, 639, K03

Kepler, S. O., et al. 2000, ApJL, 534, L185

Kleinman, S. J., et al. 1998, ApJ, 495, 424

Kotak, R., van Kerkwijk, M. H., Clemens, J. C., \& Koester, D. 2003, A\&A, 397, 1043

Kuhn, J. R. 1998, Proc. SOHO6/GONG 98 Workshop, ESA, SP-418, 871

Lenz, P., \& Breger, M. 2005, Commun. Asteroseismol., 146, 53

Li, Y., \& Yang, J. Y. 2007, MNRAS, 375, 388

Koester, D. 2008, private communication

Marchenko, S. V. 2008, A\&A, 479, 845

Matthews, J. M, et al. 2004, Nature, 430, 51

Metcalfe, T. S., Montgomery, M. H., \& Kawaler, S. D. 2003, MNRAS, 344, 88

Montgomery, M., \& Kupka, F. 2004, MNRAS, 350, 267

Montgomery, M. H. 2005, ApJ, 633, 1142

Montgomery, M. H. 2006, in ASP Conf. Ser. 352, New Horizons in Astronomy: Frank N. Bash Symp. (San Francisco, CA: ASP), 261

Montgomery, M. H. 2008a, Commun. Asteroseismol., 154, 38

Montgomery, M. H. 2008b, private communication

Morin, J., et al. 2008, MNRAS, 384, 77

Nather, R. E., \& Mukadam, A. 2004, ApJ, 605, 846

Nather, R. E., Winget, D. E., \& Clemens, J. C. 1990, ApJ, 361, 390

Prada, M., Pier, G., \& Straniero, O. 2002, ApJ, 581, 585

Provencal, J. L., et al. 2005, BAAS, 37, 1157

Thompson, S. E., Clemens, J. C., van Kerkwijk, M.H., \& Koester, D. 2003, ApJ, 589,921

Scargle, J. D. 1982, ApJ, 263, 835

Schunker, H., \& Cally, P. S. 2006, MNRAS, 372, 551

Unno, W., Osaki, Y., Ando, H, \& Shibahashi, H 1979, Nonradial Oscillations of Stars (Tokyo: Univ. Tokyo Press)

van Kerkwijk, M. H., Clemens, J. C., \& Wu, Y. 2000, MNRAS, 314, 209

Vuckovic, M., et al. 2006, ApJ, 646, 1230

Weidner, C., \& Koeter, D. 2003, A\&A, 406, 657

Winget, D. E., et al. 1990, ApJ, 357, 630

Winget, D. E., et al. 1991, ApJ, 378, 326

Winget, D. E., et al. 1994, ApJ, 430, 839, W94

Winget, D. E., Sullivan, D. J., Metcalfe, T. S., Kawaler, S. D., \& Montgomery, M. H. 2004, ApJL, 602, 109

Winget, D. E. 2008, private communication

Wood, M. 1992, ApJ, 386, 539

Woodward, M. F., \& Noyes, R. W. 1985, Nature, 449, 318

Wu, Y. 2000, ASP Conf. Ser. 203, The Impact of Large-Scale Surveys on Pulsating Star Research, IAU Colloquium 176, ed. L. Szabados \& D. Kurtz (San Francisco, CA: ASP), 508

Wu, Y. 2001, MNRAS, 323, 248

Yeates, C. M., Clemens, J. C., Thompson, S. E., \& Mullaly, F. 2005, ApJ, 635, 1239

Zola, S. 2008, private communication 